\title{
GOLDBACH REPRESENTATIONS IN ARITHMETIC PROGRESSIONS AND ZEROS OF DIRICHLET L-FUNCTIONS
}

\author{
GAUTAMI BHOWMIK, KARIN HALUPCZOK, KOHJI MATSUMOTO, AND YUTA SUZUKI
}

\begin{abstract}
Assuming a conjecture on distinct zeros of Dirichlet $L$-functions we get asymptotic results on the average number of representations of an integer as the sum of two primes in arithmetic progression. On the other hand the existence of good error terms gives information on the location of zeros of $L$-functions. Similar results are obtained for an integer in a congruence class expressed as the sum of two primes.
\end{abstract}

\section{Introduction And Results}

The Goldbach problem of representing every even integer larger than 2 as the sum of two primes has several variants, one being that in which the summands are primes in given arithmetic progressions. Similar to the original problem it is known that almost all even integers satisfying some congruence condition can be written as the sum of two primes in congruence classes. Quantitatively, the exceptional set of integers less than $X$ and satisfying the necessary congruence condition, which can not be written as the sum of primes congruent to a common modulus $q$ may be estimated as $O\left(\varphi(q)^{-1} X^{1-\delta}\right)$ for a computable positive constant $\delta$ and all $q \leq X^{\delta}$ [13]. (See [1] for more recent results.)

Though the complete solution of these binary Goldbach problems is out of sight, the related question of the average number of representations of integers as sums of primes seems more accessible. The study of the average order of the weighted function

$$
G(n)=\sum_{\ell+m=n} \Lambda(\ell) \Lambda(m)
$$

where $\Lambda$ is the von Mangoldt function has begun with Fujii [8] and continues to be actively pursued. However the current state of knowledge on the zeros of the Riemann zeta function $\zeta(s)$ is not enough to obtain "good" error terms unconditionally and the Riemann Hypothesis is always assumed in such studies. In fact obtaining sufficiently sharp error terms for average orders of the mean value of $G(n)$ is expected to solve other conjectures like the Riemann Hypothesis, as elaborated by Granville [11] in the classical case of unrestricted primes. This paper is an analogous study with the two primes in arithmetic progressions with a common modulus.

2010 Mathematics Subject Classification. 11P32, 11M26, $11 \mathrm{M} 41$.

Key words and phrases. Goldbach problem, congruences, Dirichlet $L$-function, Generalized Riemann hypothesis, Distinct Zero Conjecture, explicit formula, Siegel zero.

The first and third authors benefit from the financial support of the French-Japanese Joint Project "Zeta-functions of Several Variables and Applications" (PRC CNRS/JSPS 2015-2016). The fourth author is supported by Grant-in-Aid for JSPS Research Fellow (Grant Number: JP16J00906) and had the partial aid of CEMPI for his stay at Lille. 
The function that we consider here, with $a, b$ positive integers coprime to $q$, is

$$
G(n ; q, a, b)=\sum_{\substack{\ell+m=n \\ \ell \equiv a, m \equiv b(\bmod q)}} \Lambda(\ell) \Lambda(m)
$$

whose summatory function defined as

$$
S(x ; q, a, b)=\sum_{n \leq x} G(n ; q, a, b)
$$

was introduced by Rüppel [15] and further studied by the fourth author [16].

On the lines of Granville we consider the relations between an explicit formula for $S(x ; q, a, b)$ and zeros of $L$-functions. In $[11,1 \mathrm{~A}]$ it is stated that there is an equivalence between the estimate

$$
\sum_{n \leq x}(G(n)-J(n)) \ll x^{3 / 2+o(1)}
$$

and the Riemann Hypothesis $(\mathrm{RH})$ for $\zeta(s)$ where $J(n)=0$ for odd $n$ and, with $C_{2}=2 \prod_{p>2}\left(1-\frac{1}{(p-1)^{2}}\right)$ being the twin prime constant,

$$
J(n)=n \cdot C_{2} \prod_{\substack{p \mid n \\ p>2}} \frac{p-1}{p-2}
$$

for even $n$. The function $J(n)$ is believed since Hardy and Littlewood to be a good approximation for $G(n)$ (cf. [12]).

We denote by $\chi$ a Dirichlet character $(\bmod q)$, by $L(s, \chi)$ the associated Dirichlet $L$-function and by $\rho_{\chi}$ its non-trivial zeros. Let $B_{\chi}=\sup \left\{\Re \rho_{\chi}\right\}$ and $B_{q}=\sup \left\{B_{\chi} \mid\right.$ $\chi(\bmod q)\}$. Hence $1 / 2 \leq B_{q} \leq 1$ for $q \geq 1$. In case of the trivial character, we use $\rho$ for non-trivial zeros of $\zeta(s)$, and $B=\sup \{\Re \rho\}$.

In the context of primes in congruence classes we first need to formulate the Distinct Zero Conjecture (DZC) on zeros of $L$-functions as:

For any $q \geq 1$, any two distinct Dirichlet L-functions associated with characters of modulus $q$ do not have a common non-trivial zero, except for a possible multiple zero at $s=1 / 2$.

Though weaker than the non-coincidence conjecture found in literature that expects all zeros of all primitive $L$-functions to be linearly independent except for the possible multiple zero at $s=1 / 2$ (cf. [5, p.353]), this suffices for our purpose.

Theorem 1. Let $a, b$ be integers with $(a b, q)=1$.

(1) For $x \geq 2$, we have

$$
S(x ; q, a, b)=\frac{x^{2}}{2 \varphi(q)^{2}}+O\left(x^{1+B_{q}}\right),
$$

where the implicit constant is absolute.

(2) Let DZC be true, let $\chi(a)+\chi(b) \neq 0$ for all characters $\chi(\bmod q)$ and let $1 / 2 \leq d<1$. If the asymptotic formula

$$
S(x ; q, a, b)=\frac{x^{2}}{2 \varphi(q)^{2}}+O_{q}\left(x^{1+d+\varepsilon}\right) .
$$

holds for any $\varepsilon>0$, then $B_{q} \leq d$ or $B_{q}=1$. Further if (1.3) holds with $a=b$, then $B_{q} \leq d$. 
Remark 1. Our result thus falls short of an equivalence with the Generalized Riemann Hypothesis (GRH) for $L$-functions modulo $q$ since we have an additional possibility of $B_{q}=1$. Using a yet unpublished idea of I. Ruzsa, we were able to exclude this possibility for the case $a=b$ under the DZC .

Thus the proof of the equivalence between the $\mathrm{RH}$ and (1.1) is now complete (see also [2]). All other equivalences for primes in arithmetic progressions are still partial.

In particular if $B_{q}=1$, then the error term of Theorem 2 becomes so large that it hides the information on non-trivial zeros. Thus the analytic continuation of the generating function could only be obtained up to $\sigma>2 B_{q}$ (3).

Remark 2. We need the condition that $\chi(a)+\chi(b) \neq 0$ for all $\chi(\bmod q)$ in order to assure that the residue $r_{1}\left(\rho_{q}\right)$ in Proposition 3 does not vanish.

To prove Theorem 1 we need an explicit formula for $S(x ; q, a, b)$, which can be stated as follows.

Theorem 2. Let $a, b$ be integers with $(a b, q)=1$. Then, for $x \geq 2$ and for any $\varepsilon>0$,

$$
\begin{aligned}
& S(x ; q, a, b)=\frac{x^{2}}{2 \varphi(q)^{2}} \\
& -\frac{1}{\varphi(q)^{2}} \sum_{\chi(\bmod q)}(\overline{\chi(a)}+\overline{\chi(b)}) \sum_{\rho_{\chi}} \frac{x^{\rho_{\chi}+1}}{\rho_{\chi}\left(\rho_{\chi}+1\right)}+O\left(x^{2 B_{q}^{*}}(\log q x)^{5}\right),
\end{aligned}
$$

where the implicit constant is absolute and

$$
B_{q}^{*}=B_{q}^{*}(x)=\min \left(B_{q}, 1-\eta\right), \quad \eta=\eta_{q}(x)=\frac{c_{1}(\varepsilon)}{\min \left(q^{\varepsilon},(\log x)^{4 / 5}\right)}
$$

with some small constant $c_{1}(\varepsilon)>0$ depending only on $\varepsilon>0$.

First in Section 3 we prove an explicit formula with a weaker error estimate (Proposition 1) using a generalized Landau-Gonek formula for $L$-functions (Proposition 2 in Section 4). This weaker form is an analogue of Granville [11, Corrigendum, (2)], which states

$$
\sum_{n \leq x} G(n)=\frac{x^{2}}{2}-2 \sum_{\substack{\rho \\|\Im \rho| \leq x}} \frac{x^{\rho+1}}{\rho(\rho+1)}+O\left(x^{(2+4 B) / 3}(\log x)^{2}\right),
$$

and the proof of Proposition 1 essentially runs along the line suggested by [11]. Therefore Sections 3 and 4 include a reconstruction of Granville's argument for the asymptotic order. However we can go further; we take this opportunity to prove (in Sections 5 and 6 ) the stronger error estimate (1.4), an analogue of that announced in $[11,(5.1)]$ (cf. [11, Corrigendum, comments before (2)]), using a kind of circle method of the first author and Schlage-Puchta [3].

With the help of Theorem 2 above the analytic continuation of the Dirichlet series

$$
\sum_{n=1}^{\infty} \frac{G(n ; q, a, b)}{n^{s}}
$$

is examined in Proposition 3 (in Section 7) and this enables us to establish relations between the error terms in the average of Goldbach problems in arithmetic progressions and zeros of Dirichlet $L$-functions. 
Further, we examine the case of $n$ with modulus conditions as in $[11,1 \mathrm{~B}]$ where it is stated that the GRH for Dirichlet $L$-functions $L(s, \chi)$, over all characters $\chi$, the modulus of which are odd squarefree divisors of $q$, is equivalent to the estimate

$$
\sum_{\substack{n \leq x \\ n \equiv 2(\bmod q)}}(G(n)-J(n)) \ll x^{3 / 2+o(1)} .
$$

Moreover in $[11$, Corrigendum, $1 \mathrm{C}]$ it is stated that if the estimate

$$
\sum_{\substack{n \leq x \\ q \mid n}} G(n)=\frac{1}{\varphi(q)} \sum_{n \leq x} G(n)+O_{q}\left(x^{1+o(1)}\right)
$$

is attained then the GRH for Dirichlet $L$-functions $L(s, \chi), \chi(\bmod q)$ holds; and under this hypothesis the last estimate would have the error term $O\left(x^{4 / 3}(\log x)^{2}\right)$.

Here we extend (1.6) with the general congruence condition $n \equiv c$ for an arbitrary positive integer $c$ instead of the special case $n \equiv 2$. Assuming the GRH for $L$ functions $(\bmod q)$ we can deduce the estimate

$$
\sum_{\substack{n \leq x \\ n \equiv c(\bmod q)}}(G(n)-J(n)) \ll x^{3 / 2} .
$$

However in the other direction, we could not deduce satisfactory conclusions on the size of $B_{q}$ when $a \neq b$. In particular we were unable then to reconstruct the reverse implications for (1.6) and (1.7). In the following we give a further example of a condition with which we can get the reverse implication.

Theorem 3. Let $q, c$ be integers such that $(2, q) \mid c$.

(1) For $x \geq 2$, we have

$$
\sum_{\substack{n \leq x \\ n \equiv c(\bmod q)}}(G(n)-J(n)) \ll x^{1+B_{q}},
$$

where the implicit constant is absolute.

(2) Assume that

$$
\sum_{\substack{n \leq x \\ n \equiv c(\bmod q)}}(G(n)-J(n)) \ll_{q} x^{1+d+\varepsilon}
$$

holds for some $1 / 2 \leq d \leq 1$ and any $\varepsilon>0$. If there exists a zero $\rho_{0}$ of $\prod_{\chi(\bmod q)} L(s, \chi)$ such that

(a) $B_{q}=\Re \rho_{0}$

(b) $\rho_{0}$ belongs to a unique character $\chi_{1}(\bmod q)$

(c) the conductor $q^{*}$ of $\chi_{1}(\bmod q)$ is squarefree and satisfies $\left(c, q^{*}\right)=1$, then $B_{q}=\Re \rho_{0} \leq d$.

The conditions on $\rho_{0}$ might resemble those of the Landau-Siegel zero although (2) above is not actually applicable to a Landau-Siegel zero if there are some complex zeros of $L$-function of the same modulus which are very close to the vertical line $\sigma=1$. 
To obtain the above results we require an asymptotic formula with $B_{q}^{*}$ as in Theorem 2 .

Theorem 4. For $x \geq 2, \varepsilon>0$ and for any positive integer $c$ we have

$$
\begin{array}{r}
\sum_{\substack{n \leq x \\
n \equiv c(\bmod q)}} G(n)=\frac{\mathfrak{S}_{q}(c)}{2} x^{2}-\frac{2}{\varphi(q)^{2}} \sum_{\substack{a=1 \\
(a(c-a), q)=1}}^{q} \sum_{\chi(\bmod q)} \overline{\chi(a)} \sum_{\rho_{\chi}} \frac{x^{\rho_{\chi}+1}}{\rho_{\chi}\left(\rho_{\chi}+1\right)} \\
+O\left(x^{\left.2 B_{q}^{*}(\log q x)^{5}\right),}\right.
\end{array}
$$

where

$$
\mathfrak{S}_{q}(c)=\frac{1}{\varphi(q)} \prod_{\substack{p \mid q \\ p \nmid c}} \frac{p-2}{p-1},
$$

and the implicit constant is absolute.

Note that $\mathfrak{S}_{q}(c)=0$ if $(2, q) \nmid c$. The above theorem is proven in Section 6 . To ensure the uniformity of $q$ it is not enough to sum Theorem 2 over residues and we need other tools like Lemma 10 . Finally, using Theorem 4, we give the proof of (1.8) and Theorem 3 in Section 7. In Section 8, we give the proof for the supplement of Theorem $1(2)$ in the case $a=b$.

Acknowledgements. The first two authors are grateful to Professor Andrew Granville for helpful discussions. Thanks are due to Professor Masatoshi Suzuki for useful information, and to Professor Keiju Sono and the referee for their valuable comments on the first version of the manuscript. We particularly thank Professor Imre Ruzsa for the idea that improves Theorem 1 (2) in the case $a=b$.

\section{Some PRELIMINARIES}

In this section we fix notation on the zeros of Dirichlet $L$-functions and give some basic lemmas on Dirichlet $L$-functions. Results used directly from [14] are only cited but in other cases details are added .

As we mentioned in Section 1, we denote by $\chi(\bmod q)$ a Dirichlet character $(\bmod q)$ and by $\chi^{*}\left(\bmod q^{*}\right)$ the primitive character inducing $\chi(\bmod q)$. If there is no specific mention, any statement with $\chi(\bmod q)$ is stated for any $q \geq 1$ and any character $\chi(\bmod q)$.

We denote the Dirichlet $L$-function associated to $\chi(\bmod q)$ by $L(s, \chi)$. We say a zero of $L(s, \chi)$ is non-trivial, if it is contained in the strip $0<\sigma<1$. We denote by $\rho_{\chi}$ non-trivial zeros of $L(s, \chi)$ with the real part $\beta_{\chi}$ and the imaginary part $\gamma_{\chi}$. As a summation variable, the letter $\rho_{\chi}$ runs through all non-trivial zeros of $L(s, \chi)$ counted with multiplicity. We denote the Landau-Siegel zero of $(\bmod q)$ by $\beta_{1}$.

We let $\delta_{0}(\chi)=1$ if $\chi=\chi_{0}$ is the principal character and $\delta_{0}(\chi)=0$ otherwise. Similarly we let $\delta_{1}(\chi)=1$ if $\chi$ is the exceptional character (that is, whose $L$-function has a Landau-Siegel zero) and $\delta_{1}(\chi)=0$ otherwise.

We first evoke some lemmas for sums over non-trivial zeros.

Lemma 1 ([14, Theorem 10.17]). For any $T \geq 0$, we have

$$
\sum_{\substack{\rho_{\chi} \\ T \leq\left|\gamma_{\chi}\right| \leq T+1}} 1 \ll \log q(|T|+2) .
$$


Lemma 2. For any $T \geq 1$ and $\chi(\bmod q)$, we have

$$
\sum_{\substack{\rho_{\chi} \neq 1-\beta_{1} \\\left|\gamma_{\chi}\right| \leq T}} \frac{1}{\left|\rho_{\chi}\right|} \ll(\log 2 q T)^{2}, \quad \sum_{\substack{\rho_{\chi} \\\left|\gamma_{\chi}\right| \leq T}} \frac{1}{\left|\rho_{\chi}\right|} \ll(\log 2 q T)^{2}+\delta_{1}(\chi) q^{1 / 2}(\log q)^{2} .
$$

Proof. For the first estimate we dissect the sum at $\left|\gamma_{\chi}\right|=1$ as

$$
\sum_{\substack{\rho_{\chi} \neq 1-\beta_{1} \\\left|\gamma_{\chi}\right| \leq T}} \frac{1}{\left|\rho_{\chi}\right|}=\sum_{\substack{\rho_{\chi} \neq 1-\beta_{1} \\\left|\gamma_{\chi}\right| \leq 1}} \frac{1}{\left|\rho_{\chi}\right|}+\sum_{\substack{\rho_{\chi} \\ 1<\left|\gamma_{\chi}\right| \leq T}} \frac{1}{\left|\rho_{\chi}\right|}
$$

For the first sum Lemma 1 gives

$$
\begin{aligned}
\sum_{\substack{\rho_{\chi} \neq 1-\beta_{1} \\
\left|\gamma_{\chi}\right| \leq 1}} \frac{1}{\left|\rho_{\chi}\right|} \leq \sum_{\substack{\rho_{\chi} \neq 1-\beta_{1} \\
\left|\gamma_{\chi}\right| \leq 1}} \frac{1}{\beta_{\chi}}=\sum_{\substack{\rho_{\chi} \neq \beta_{1} \\
\left|\gamma_{\chi}\right| \leq 1}} \frac{1}{1-\beta_{\chi}} \\
\ll \sum_{\substack{\rho_{\chi} \\
\left|\gamma_{\chi}\right| \leq 1 \\
\beta_{\chi}>1-c_{0} / \log 2 q}} \frac{1}{1-\beta_{\chi}} \ll(\log 2 q) \sum_{\substack{\rho_{\chi} \\
\left|\gamma_{\chi}\right| \leq 1}} 1 \ll(\log 2 q)^{2} \ll(\log 2 q T)^{2},
\end{aligned}
$$

where $c_{0}>0$ is some small absolute constant while for the second sum, Lemma 1 gives

$$
\sum_{\substack{\rho_{\chi} \\ 1<\left|\gamma_{\chi}\right| \leq T}} \frac{1}{\left|\rho_{\chi}\right|} \leq \sum_{n \leq T} \sum_{\substack{\rho_{\chi} \\ n<\left|\gamma_{\chi}\right| \leq n+1}} \frac{1}{\left|\rho_{\chi}\right|} \ll \sum_{n \leq T} \frac{\log q(n+2)}{n} \ll(\log 2 q T)^{2} .
$$

Thus the first estimate follows. The second estimate is obtained by the first estimate combined with the well-known bound [14, Corollary 11.12] of the Siegel zero

$$
\beta_{1}>1-\frac{c_{2}}{q^{1 / 2}(\log q)^{2}}
$$

where $c_{2}>0$ is some absolute constant.

Lemma 3. For any $T \geq 1$ and $\chi(\bmod q)$, we have

$$
\sum_{\substack{\rho_{\chi} \\\left|\rho_{\chi}\right|>T}} \frac{1}{\left|\rho_{\chi}\right|^{2}} \ll \frac{\log 2 q T}{T}, \quad \sum_{\rho_{\chi}} \frac{1}{\left|\rho_{\chi}\left(\rho_{\chi}+1\right)\right|} \ll(\log 2 q)^{2}+\delta_{1}(\chi) q^{1 / 2}(\log q)^{2} .
$$

Proof. The first estimate is again obtained by using Lemma 1 ,i.e.

$$
\sum_{\substack{\rho_{\chi} \\\left|\gamma_{\chi}\right|>T}} \frac{1}{\left|\rho_{\chi}\right|^{2}} \leq \sum_{n=[T]}^{\infty} \sum_{\substack{\rho_{\chi} \\ n<\left|\gamma_{\chi}\right| \leq n+1}} \frac{1}{\left|\rho_{\chi}\right|^{2}} \ll \sum_{n=[T]}^{\infty} \frac{\log q n}{n^{2}} \ll \frac{\log q T}{T},
$$

whereas the last estimate can be obtained by comparison to an integral. For the latter estimate, we combine the former one with Lemma 2. This gives

$$
\sum_{\rho_{\chi}} \frac{1}{\left|\rho_{\chi}\left(\rho_{\chi}+1\right)\right|} \leq \sum_{\substack{\rho_{\chi} \\\left|\gamma_{\chi}\right| \leq 1}} \frac{1}{\left|\rho_{\chi}\right|}+\sum_{\substack{\rho_{\chi} \\\left|\gamma_{\chi}\right|>1}} \frac{1}{\left|\rho_{\chi}\right|^{2}} \ll(\log 2 q)^{2}+\delta_{1}(\chi) q^{1 / 2}(\log q)^{2} .
$$


We next prepare some explicit formulas for the sum

$$
\psi(x, \chi)=\sum_{n \leq x} \chi(n) \Lambda(n) .
$$

The next result appears in [14] with the restriction that $\chi(\bmod q)$ be primitive.

Lemma 4. For any $u, T \geq 2$, the explicit formula

$$
\psi(u, \chi)=\delta_{0}(\chi) u-\sum_{\substack{\rho_{\chi} \\\left|\gamma_{\chi}\right| \leq T}} \frac{u^{\rho_{\chi}}}{\rho_{\chi}}+C\left(\chi^{*}\right)+E(u, T, \chi)
$$

holds, where

$$
E(u, T, \chi) \ll(\log 2 q)(\log u)+\frac{u}{T}(\log q u T)^{2}
$$

and $C(\chi)$ is some constant depending only on $\chi$.

Proof. For primitive $\chi$, this follows immediately from Theorem 12.5 and 12.10 of [14] as below. If $\chi$ is trivial so that $q=1$, we use Theorem 12.5 of [14] with $x=u$. We can estimate the last three terms on the right-hand side of (12.3) of [14] as

$$
-\log 2 \pi-\frac{1}{2} \log \left(1-1 / u^{2}\right)+R(u, T) \ll(\log 2 q)(\log u)+\frac{u}{T}(\log q u T)^{2},
$$

by using (12.4) of [14]. This gives the assertion for the case when $\chi$ is trivial. If $\chi$ is non-principal, we use Theorem 12.10 of [14] with $x=u$. The last four terms on the right-hand side of (12.6) of [14] can be rewritten as

$$
\begin{gathered}
-\frac{1}{2} \log (u-1)-\frac{\chi(-1)}{2} \log (u+1)+C(\chi)+R(u, T ; \chi) \\
=C(\chi)+O\left((\log 2 q)(\log u)+\frac{u}{T}(\log q u T)^{2}\right) .
\end{gathered}
$$

This gives the assertion for the case of $\chi$ being primitive and non-principal.

If $\chi$ is imprimitive, then it suffices to note that the non-trivial zeros of $L(s, \chi)$ are those of $L\left(s, \chi^{*}\right)$ and that

$$
\begin{aligned}
\psi(u, \chi)-\psi\left(u, \chi^{*}\right) \ll \sum_{\substack{n \leq u \\
(n, q)>1}} \Lambda(n) & =\sum_{p \mid q}(\log p)\left[\frac{\log u}{\log p}\right] \\
& \leq\left(\frac{\log u}{\log 2}\right) \sum_{p \mid q}(\log p) \leq(\log 2 q)(\log u),
\end{aligned}
$$

which is absorbed into $E(u, T, \chi)$.

When we substitute the above explicit formula into some sum or integral, we need to use a uniform parameter $T$ and a uniform bound of the error term. Also it is convenient to work with the case $0 \leq u<2$. Thus we modify the above explicit formula in the following form.

Lemma 5. For any $x \geq T \geq 2$ and $x \geq u \geq 0$, the explicit formula

$$
\psi(u, \chi)=\delta_{0}(\chi) u-\sum_{\substack{\rho_{\chi} \\\left|\gamma_{\chi}\right| \leq T}} \frac{u^{\rho_{\chi}}}{\rho_{\chi}}+O\left(\frac{x}{T}(\log q x)^{2}+\delta_{1}(\chi) q^{1 / 2}(\log q)^{2}\right)
$$

holds. 
Proof. We first consider the case $u \geq 2$. For this we use Lemma 4. Since $u \leq x \leq T$, the error term of Lemma 4 is

$$
E(u, T, \chi) \ll(\log 2 q)(\log x)+\frac{x}{T}(\log q x T)^{2} \ll \frac{x}{T}(\log q x T)^{2} .
$$

Further, by Theorem 11.4 of [14], we know that $\left(L^{\prime} / L\right)\left(1, \overline{\chi^{*}}\right) \ll \log 2 q$ if $\chi$ is not exceptional, while for the exceptional $\chi$ and exceptional zero $\beta_{1},(2.1)$ gives

$$
\frac{L^{\prime}}{L}\left(1, \overline{\chi^{*}}\right)=\frac{1}{1-\beta_{1}}+O(\log 2 q) \ll q^{1 / 2}(\log q)^{2} .
$$

Therefore by (12.7) of [14], we obtain

$$
C\left(\chi^{*}\right) \ll \log 2 q+\delta_{1}(\chi) q^{1 / 2}(\log q)^{2} \ll \frac{x}{T}(\log q x)^{2}+\delta_{1}(\chi) q^{1 / 2}(\log q)^{2}
$$

for non-principal $\chi$, from which the lemma follows for the case $u \geq 2$.

The remaining case is when $0 \leq u<2$. Now the sum on the right-hand side of (2.3) is estimated by using Lemma 2 as

$$
\begin{aligned}
\sum_{\substack{\rho_{\chi} \\
\left|\gamma_{\chi}\right| \leq T}} \frac{u^{\rho_{\chi}}}{\rho_{\chi}} \ll u \sum_{\substack{\rho_{\chi} \\
\left|\gamma_{\chi}\right| \leq T}} \frac{1}{\left|\rho_{\chi}\right|} & \ll(\log q T)^{2}+\delta_{1}(\chi) q^{1 / 2}(\log q)^{2} \\
& \ll \frac{x}{T}(\log q x)^{2}+\delta_{1}(\chi) q^{1 / 2}(\log q)^{2} .
\end{aligned}
$$

since $T \leq x$. On the other hand, when $u<2$, the left-hand side of (2.3) is zero. Therefore the assertion holds trivially.

\section{An ASYmptotic Formula for $S(x ; q, a, b)$}

In this section, we deduce a prototype of Theorem 2 along the line of [11]:

Proposition 1. For integers $a, b, q$ with $(a b, q)=1$, we have

$$
\begin{aligned}
S(x ; q, a, b)=\frac{x^{2}}{2 \varphi(q)^{2}}-\frac{1}{\varphi(q)^{2}} \sum_{\chi(\bmod q)}(\overline{\chi(a)}+\overline{\chi(b)}) & \sum_{\rho_{\chi}} \frac{x^{\rho_{\chi}+1}}{\rho_{\chi}\left(\rho_{\chi}+1\right)} \\
& +O\left(x^{\left(2+4 B_{q}\right) / 3}(\log q x)^{4}\right),
\end{aligned}
$$

where the implicit constant is absolute.

The proof is divided into three parts.

Step 1: The first substitution in the explicit formula.

With

$$
\psi(x ; q, a)=\sum_{\substack{m \leq x \\ m \equiv a(\bmod q)}} \Lambda(m),
$$

we can write

$$
S(x ; q, a, b)=\sum_{\substack{\ell \leq x \\ \ell \equiv a(\bmod q)}} \Lambda(\ell) \psi(x-\ell ; q, b) .
$$

Using the orthogonality relation of Dirichlet characters, we have

$$
\psi(x ; q, b)=\frac{1}{\varphi(q)} \sum_{\chi(\bmod q)} \overline{\chi(b)} \psi(x, \chi) .
$$


We substitute the explicit formula given by Lemma 5 here. This gives

$$
\psi(u ; q, b)=\frac{1}{\varphi(q)} A(u, T ; q, b)+B(u, T ; q, b)
$$

for $x \geq T \geq 2$ and $x \geq u \geq 0$, where

$$
A(u, T ; q, b)=u-\sum_{\chi(\bmod q)} \overline{\chi(b)} \sum_{\substack{\rho_{\chi} \\\left|\gamma_{\chi}\right| \leq T}} \frac{u^{\rho_{\chi}}}{\rho_{\chi}}
$$

and $B(u, T ; q, b)$ is the error term satisfying

$$
B(u, T ; q, b) \ll \frac{x}{T}(\log q x)^{2}+\frac{1}{\varphi(q)} \sum_{\chi(\bmod q)} \delta_{1}(\chi) q^{1 / 2}(\log q)^{2} \ll \frac{x}{T}(\log q x)^{2},
$$

where for estimating the term involving $\delta_{1}(\chi) q^{1 / 2}(\log q)^{2}$, we use the fact that there is at most one exceptional character $(\bmod q)$. Substituting (3.2) into (3.1), we obtain

$$
S(x ; q, a, b)=\frac{1}{\varphi(q)} \sum_{\substack{\ell \leq x \\ \ell \equiv a(\bmod q)}} \Lambda(\ell) A(x-\ell, T ; q, b)+O\left(\frac{x^{2}}{T}(\log q x)^{2}\right) .
$$

Step 2: The second substitution in the explicit formula.

Now we evaluate the sum on the right-hand side of (3.3), we split it into two parts.

$$
\begin{aligned}
& \frac{1}{\varphi(q)} \sum_{\substack{\ell \leq x \\
\ell \equiv a(\bmod q)}} \Lambda(\ell) A(x-\ell, T ; q, b) \\
= & \frac{1}{\varphi(q)} \sum_{\substack{\ell \leq x \\
\ell \equiv a(\bmod q)}} \Lambda(\ell)(x-\ell) \\
& -\frac{1}{\varphi(q)} \sum_{\substack{\ell \leq x \\
\ell \equiv a(\bmod q)}} \Lambda(\ell) \sum_{\chi(\bmod q)} \overline{\chi(b)} \sum_{\substack{\rho_{\chi} \\
\left|\gamma_{\chi}\right| \leq T}} \frac{(x-\ell)^{\rho_{\chi}}}{\rho_{\chi}} \\
= & \Sigma_{1}-\Sigma_{2}, \text { say. }
\end{aligned}
$$

Consider $\Sigma_{1}$. We have

$$
\Sigma_{1}=\frac{1}{\varphi(q)} \sum_{\substack{\ell \leq x \\ \ell \equiv a(\bmod q)}} \Lambda(\ell) \int_{\ell}^{x} d u=\frac{1}{\varphi(q)} \int_{0}^{x} \psi(u ; q, a) d u,
$$

so, using (3.2), we obtain

$$
\Sigma_{1}=\frac{1}{\varphi(q)^{2}} \int_{0}^{x} A(u, T ; q, a) d u+O\left(\frac{x^{2}}{T}(\log q x)^{2}\right) .
$$

Inserting the definition of $A(u, T ; q, a)$ yields

$$
\Sigma_{1}=\frac{1}{\varphi(q)^{2}}\left(\frac{x^{2}}{2}-\sum_{\chi(\bmod q)} \overline{\chi(a)} \sum_{\substack{\rho_{\chi} \\\left|\gamma_{\chi}\right| \leq T}} \frac{x^{\rho_{\chi}+1}}{\rho_{\chi}\left(\rho_{\chi}+1\right)}\right)+O\left(\frac{x^{2}}{T}(\log q x)^{2}\right) .
$$


10 GAUTAMI BHOWMIK, KARIN HALUPCZOK, KOHJI MATSUMOTO, AND YUTA SUZUKI

Next consider $\Sigma_{2}$. We have

$$
\Sigma_{2}=\frac{1}{\varphi(q)} \sum_{\chi(\bmod q)} \overline{\chi(b)} \sum_{\substack{\rho_{\chi} \\\left|\gamma_{\chi}\right| \leq T}} \Psi\left(\rho_{\chi}, x ; q, a\right)
$$

where

$$
\Psi\left(\rho_{\chi}, x ; q, a\right)=\frac{1}{\rho_{\chi}} \sum_{\substack{\ell \leq x \\ \ell \equiv a(\bmod q)}} \Lambda(\ell)(x-\ell)^{\rho_{\chi}} .
$$

Again using (3.2),

$$
\begin{aligned}
\Psi\left(\rho_{\chi}, x ; q, a\right)= & \frac{1}{\rho_{\chi}} \int_{0}^{x}(x-u)^{\rho_{\chi}} d \psi(u ; q, a) \\
= & \frac{1}{\rho_{\chi}} \int_{0}^{x}(x-u)^{\rho_{\chi}}\left(\frac{1}{\varphi(q)} d A(u, T ; q, a)+d B(u, T ; q, a)\right) \\
= & \frac{1}{\varphi(q) \rho_{\chi}} \int_{0}^{x}(x-u)^{\rho_{\chi}} d u \\
& \quad-\frac{1}{\varphi(q) \rho_{\chi}} \sum_{\chi^{\prime}(\bmod q)} \frac{1}{\chi^{\prime}(a)} \sum_{\rho_{\chi^{\prime}}} \int_{0}^{x}(x-u)^{\rho_{\chi}} u^{\rho_{\chi^{\prime}}-1} d u \\
& \quad+\frac{1}{\rho_{\chi}} \int_{0}^{x}(x-u)^{\rho_{\chi}} d B(u, T ; q, a) \\
= & J_{1}-J_{2}+J_{3}, \text { say. }
\end{aligned}
$$

Obviously

Since

$$
J_{1}=\frac{x^{\rho_{\chi}+1}}{\varphi(q) \rho_{\chi}\left(\rho_{\chi}+1\right)}
$$

$$
\int_{0}^{x}(x-u)^{\rho_{\chi}} u^{\rho_{\chi^{\prime}}-1} d u=x^{\rho_{\chi}+\rho_{\chi^{\prime}}} \frac{\rho_{\chi} \Gamma\left(\rho_{\chi}\right) \Gamma\left(\rho_{\chi^{\prime}}\right)}{\left(\rho_{\chi}+\rho_{\chi^{\prime}}\right) \Gamma\left(\rho_{\chi}+\rho_{\chi^{\prime}}\right)}
$$

we have

$$
J_{2}=\frac{1}{\varphi(q)} \sum_{\chi^{\prime}(\bmod q)} \overline{\chi^{\prime}(a)} \sum_{\substack{\rho_{\chi^{\prime}} \\\left|\gamma_{\chi^{\prime}}\right| \leq T}} \mathcal{Z}\left(\rho_{\chi}, \rho_{\chi^{\prime}}\right) x^{\rho_{\chi}+\rho_{\chi^{\prime}}}
$$

where

$$
\mathcal{Z}\left(\rho_{\chi}, \rho_{\chi^{\prime}}\right)=\frac{\Gamma\left(\rho_{\chi}\right) \Gamma\left(\rho_{\chi^{\prime}}\right)}{\left(\rho_{\chi}+\rho_{\chi^{\prime}}\right) \Gamma\left(\rho_{\chi}+\rho_{\chi^{\prime}}\right)}=\frac{\Gamma\left(\rho_{\chi}\right) \Gamma\left(\rho_{\chi^{\prime}}\right)}{\Gamma\left(1+\rho_{\chi}+\rho_{\chi^{\prime}}\right)}
$$

Lastly,

$$
\begin{aligned}
J_{3} & =\frac{1}{\rho_{\chi}}\left[(x-u)^{\rho_{\chi}} B(u, T ; q, a)\right]_{u=0}^{x}+\int_{0}^{x}(x-u)^{\rho_{\chi}-1} B(u, T ; q, a) d u \\
& =O\left(\frac{1}{\left|\rho_{\chi}\right|} \frac{x^{2}}{T}(\log q x)^{2}\right)+\int_{0}^{x}(x-u)^{\rho_{\chi}-1} B(u, T ; q, a) d u .
\end{aligned}
$$

Therefore we now obtain

$$
\Psi\left(\rho_{\chi}, x ; q, a\right)=\frac{x^{\rho_{\chi}+1}}{\varphi(q) \rho_{\chi}\left(\rho_{\chi}+1\right)}-\frac{1}{\varphi(q)} \sum_{\chi^{\prime}(\bmod q)} \overline{\chi^{\prime}(a)} \sum_{\substack{\rho_{\chi^{\prime}} \\\left|\gamma_{\chi^{\prime}}\right| \leq T}} \mathcal{Z}\left(\rho_{\chi}, \rho_{\chi^{\prime}}\right) x^{\rho_{\chi}+\rho_{\chi^{\prime}}}
$$




$$
+\int_{0}^{x} u^{\rho_{\chi}-1} B(x-u, T ; q, a) d u+O\left(\frac{1}{\left|\rho_{\chi}\right|} \frac{x^{2}}{T}(\log q x)^{2}\right) .
$$

Substituting this into (3.7) and using Lemma 2, we obtain

$$
\Sigma_{2}=\frac{1}{\varphi(q)^{2}} \sum_{\chi(\bmod q)} \overline{\chi(b)} \sum_{\substack{\rho_{\chi} \\\left|\gamma_{\chi}\right| \leq T}} \frac{x^{\rho_{\chi}+1}}{\rho_{\chi}\left(\rho_{\chi}+1\right)}-\Sigma_{3}+\Sigma_{4}+O\left(\frac{x^{2}}{T}(\log q x)^{4}\right) .
$$

where

$$
\begin{aligned}
& \Sigma_{3}=\frac{1}{\varphi(q)^{2}} \sum_{\chi(\bmod q)} \overline{\chi(b)} \sum_{\chi^{\prime}(\bmod q)} \overline{\chi^{\prime}(a)} \sum_{\substack{\rho_{\chi} \\
\left|\gamma_{\chi}\right| \leq T}} \sum_{\substack{\rho_{\chi^{\prime}} \\
\gamma_{\chi^{\prime}} \mid \leq T}} \mathcal{Z}\left(\rho_{\chi}, \rho_{\chi^{\prime}}\right) x^{\rho_{\chi}+\rho_{\chi^{\prime}},}, \\
& \Sigma_{4}=\frac{1}{\varphi(q)} \sum_{\chi(\bmod q)} \overline{\chi(b)} \sum_{\substack{\rho_{\chi} \\
\left|\gamma_{\chi}\right| \leq T}} \int_{0}^{x} u^{\rho_{\chi}-1} B(x-u, T ; q, a) d u .
\end{aligned}
$$

Combining (3.3) with (3.4) yields

$$
S(x ; q, a, b)=\Sigma_{1}-\Sigma_{2}+O\left(\frac{x^{2}}{T}(\log q x)^{2}\right),
$$

so with (3.6) and (3.9) we now arrive at

$$
\begin{aligned}
S(x ; q, a, b)=\frac{x^{2}}{2 \varphi(q)^{2}}-\frac{1}{\varphi(q)^{2}} \sum_{\chi(\bmod q)}(\overline{\chi(a)} & +\overline{\chi(b)}) \sum_{\substack{\rho_{\chi} \\
\left|\gamma_{\chi}\right| \leq T}} \frac{x^{\rho_{\chi}+1}}{\rho_{\chi}\left(\rho_{\chi}+1\right)} \\
& +\Sigma_{3}-\Sigma_{4}+O\left(\frac{x^{2}}{T}(\log q x)^{4}\right) .
\end{aligned}
$$

We next extend the sum over zeros. By Lemma 3, we have

$$
\sum_{\substack{\rho_{\chi} \\\left|\gamma_{\chi}\right|>T}} \frac{x^{\rho_{\chi}+1}}{\rho_{\chi}\left(\rho_{\chi}+1\right)} \ll x^{2} \sum_{\substack{\rho_{\chi} \\\left|\gamma_{\chi}\right|>T}} \frac{1}{\left|\gamma_{\chi}\right|^{2}} \ll \frac{x^{2}}{T}(\log q x) .
$$

Therefore we can extend the sum over zeros as

$$
\begin{aligned}
S(x ; q, a, b)=\frac{x^{2}}{2 \varphi(q)^{2}}-\frac{1}{\varphi(q)^{2}} \sum_{\chi(\bmod q)}(\overline{\chi(a)}+\overline{\chi(b)}) \sum_{\rho_{\chi}} \frac{x^{\rho_{\chi}+1}}{\rho_{\chi}\left(\rho_{\chi}+1\right)} \\
+\Sigma_{3}-\Sigma_{4}+O\left(\frac{x^{2}}{T}(\log q x)^{4}\right) .
\end{aligned}
$$

Step 3: The estimation of $\Sigma_{3}$ and $\Sigma_{4}$.

Lastly we estimate the remaining error terms $\Sigma_{3}$ and $\Sigma_{4}$.

First consider $\Sigma_{4}$. The contribution of the integral on the interval $0 \leq u \leq 3$ is

$$
\begin{aligned}
& \ll \frac{1}{\varphi(q)} \frac{x}{T}(\log q x)^{2} \sum_{\chi(\bmod q)} \sum_{\substack{\rho_{\chi} \\
\left|\gamma_{\chi}\right| \leq T}} \int_{0}^{3} u^{\beta_{\chi}-1} d u \\
& \ll \frac{1}{\varphi(q)} \frac{x}{T}(\log q x)^{2} \sum_{\chi(\bmod q)} \sum_{\substack{\rho_{\chi} \\
\left|\gamma_{\chi}\right| \leq T}} \frac{1}{\beta_{\chi}}
\end{aligned}
$$


12 GAUTAMI BHOWMIK, KARIN HALUPCZOK, KOHJI MATSUMOTO, AND YUTA SUZUKI

$$
\begin{aligned}
& \ll \frac{1}{\varphi(q)} \frac{x}{T}(\log q x)^{2} \sum_{\chi(\bmod q)} \sum_{\substack{\rho_{\chi} \neq 1-\beta_{1} \\
\left|\gamma_{\chi}\right| \leq T}} \frac{1}{\beta_{\chi}}+\frac{q^{1 / 2}}{\varphi(q)} \frac{x}{T}(\log q x)^{4} \\
& \ll \frac{1}{\varphi(q)} \frac{x}{T}(\log q x)^{2} \sum_{\chi(\bmod q)} \sum_{\substack{\rho_{\chi} \\
\left|\gamma_{\chi}\right| \leq T \\
\beta_{\chi}>1-c_{0} / \log q T}} \frac{1}{\beta_{\chi}}+\frac{x}{T}(\log q x)^{4} \\
& \ll \frac{1}{\varphi(q)} \frac{x}{T}(\log q x)^{3} \sum_{\chi(\bmod q)} \sum_{\substack{\rho_{\chi} \\
\left|\gamma_{\chi}\right| \leq T}} 1+\frac{x}{T}(\log q x)^{4} \ll x(\log q x)^{4}
\end{aligned}
$$

by (2.1) provided $T \leq x$, so we have

$$
\begin{aligned}
\Sigma_{4}=\frac{1}{\varphi(q)} \sum_{\chi(\bmod q)} \overline{\chi(b)} \int_{3}^{x}\left(\sum_{\substack{\rho_{\chi} \\
\left|\gamma_{\chi}\right| \leq T}} u^{\rho_{\chi}-1}\right) B(x-u, T ; q, a) d u & +O\left(x(\log q x)^{4}\right) .
\end{aligned}
$$

If $3 \leq u \leq x$ and $T \leq x$, then Proposition 2, proven in the next section, yields

$$
\sum_{\substack{\rho_{\chi} \\\left|\gamma_{\chi}\right| \leq T}} u^{\rho_{\chi}} \ll u \log (q u T) \log \log u+T \log u \ll u(\log q x)^{2}+x(\log q x) .
$$

Note that Proposition 2 is stated for primitive $\chi$, but the above estimate is valid for any $\chi$. Using this estimate from (3.12) we obtain

$$
\Sigma_{4} \ll \frac{x^{2}}{T}(\log q x)^{4}
$$

if $T \leq x$.

Next consider $\Sigma_{3}$. The following argument is inspired by [11, Corrigendum], but in our case we have to treat the zeros near the real line more carefully since $\Gamma(s)$ has a simple pole at $s=0$. We evaluate $\mathcal{Z}\left(\rho_{\chi}, \rho_{\chi^{\prime}}\right)$ defined in (3.8) for $\left|\gamma_{\chi}\right| \leq\left|\gamma_{\chi^{\prime}}\right|$ by using Stirling's formula

$$
\Gamma(s) \ll(|t|+1)^{\sigma-1 / 2} e^{-(\pi / 2)|t|}, \quad s=\sigma+i t, 0 \leq \sigma \leq 3,|t| \geq 1 .
$$

If $\left|\gamma_{\chi}\right| \leq\left|\gamma_{\chi^{\prime}}\right| \leq 1$, then $\left|\Gamma\left(1+\rho_{\chi}+\rho_{\chi^{\prime}}\right)\right| \asymp 1$, and hence

$$
\mathcal{Z}\left(\rho_{\chi}, \rho_{\chi^{\prime}}\right) \ll\left|\rho_{\chi}\right|^{-1}\left|\rho_{\chi^{\prime}}\right|^{-1} \ll T^{1 / 2}\left|\rho_{\chi}\right|^{-1}\left|\rho_{\chi^{\prime}}\right|^{-1} \text {. }
$$

If $\left|\gamma_{\chi}\right| \leq 1 \leq\left|\gamma_{\chi^{\prime}}\right|$, then applying Stirling's formula to $\Gamma\left(\rho_{\chi^{\prime}}\right)$ and $\Gamma\left(1+\rho_{\chi}+\rho_{\chi^{\prime}}\right)$,

$$
\begin{aligned}
\mathcal{Z}\left(\rho_{\chi}, \rho_{\chi^{\prime}}\right) & \ll\left|\rho_{\chi}\right|^{-1} \frac{\left|\gamma_{\chi^{\prime}}\right|^{\beta_{\chi^{\prime}}-1 / 2} e^{-(\pi / 2)\left|\gamma_{\chi^{\prime}}\right|}}{\left(\left|\gamma_{\chi}+\gamma_{\chi^{\prime}}\right|+1\right)^{\beta_{\chi}+\beta_{\chi^{\prime}}+1 / 2} e^{-(\pi / 2)\left|\gamma_{\chi}+\gamma_{\chi^{\prime}}\right|}} \\
& \ll\left|\rho_{\chi}\right|^{-1}\left|\gamma_{\chi^{\prime}}\right|^{-\beta_{\chi}-1} \ll\left|\rho_{\chi}\right|^{-1}\left|\rho_{\chi^{\prime}}\right|^{-1} \ll T^{1 / 2}\left|\rho_{\chi}\right|^{-1}\left|\rho_{\chi^{\prime}}\right|^{-1}
\end{aligned}
$$

as in the case $\left|\gamma_{\chi}\right| \leq\left|\gamma_{\chi^{\prime}}\right| \leq 1$. If $1 \leq\left|\gamma_{\chi}\right| \leq\left|\gamma_{\chi^{\prime}}\right| \leq T$, we have

$$
\mathcal{Z}\left(\rho_{\chi}, \rho_{\chi^{\prime}}\right) \ll \frac{\left|\gamma_{\chi}\right|^{\beta_{\chi}-1 / 2} e^{-(\pi / 2)\left|\gamma_{\chi}\right|}\left|\gamma_{\chi^{\prime}}\right|^{\beta_{\chi^{\prime}}-1 / 2} e^{-(\pi / 2)\left|\gamma_{\chi^{\prime}}\right|}}{\left(\left|\gamma_{\chi}+\gamma_{\chi^{\prime}}\right|+1\right)^{\beta_{\chi}+\beta_{\chi^{\prime}}+1 / 2} e^{-(\pi / 2)\left|\gamma_{\chi}+\gamma_{\chi^{\prime}}\right|}} .
$$

When $\gamma_{\chi}$ and $\gamma_{\chi^{\prime}}$ have the same sign, then the exponential factors are cancelled and we obtain

$$
\mathcal{Z}\left(\rho_{\chi}, \rho_{\chi^{\prime}}\right) \ll\left|\gamma_{\chi}\right|^{\beta_{\chi}-1 / 2}\left|\gamma_{\chi^{\prime}}\right|^{-\beta_{\chi}-1} \ll\left|\gamma_{\chi}\right|^{-1 / 2}\left|\gamma_{\chi^{\prime}}\right|^{-1} \ll T^{1 / 2}\left|\gamma_{\chi}\right|^{-1}\left|\gamma_{\chi^{\prime}}\right|^{-1}
$$


since $\left|\gamma_{\chi}\right| \leq\left|\gamma_{\chi^{\prime}}\right| \leq T$. When they have opposite signs the contribution of the exponential factors is $O\left(e^{-\pi\left|\gamma_{\chi}\right|}\right)$, and

$$
\begin{aligned}
& \left(\left|\gamma_{\chi}+\gamma_{\chi^{\prime}}\right|+1\right)^{-\left(\beta_{\chi}+\beta_{\chi^{\prime}}+1 / 2\right)} \\
= & \left(1+\left|\gamma_{\chi^{\prime}}\right|-\left|\gamma_{\chi}\right|\right)^{-\left(\beta_{\chi}+\beta_{\chi^{\prime}}+1 / 2\right)} \\
= & \left(1+\left|\gamma_{\chi^{\prime}}\right|\right)^{-\left(\beta_{\chi}+\beta_{\chi^{\prime}}+1 / 2\right)}\left(1-\frac{\left|\gamma_{\chi}\right|}{1+\left|\gamma_{\chi^{\prime}}\right|}\right)^{-\left(\beta_{\chi}+\beta_{\chi^{\prime}}+1 / 2\right)} \\
\leq & \left(1+\left|\gamma_{\chi^{\prime}}\right|\right)^{-\left(\beta_{\chi}+\beta_{\chi^{\prime}}+1 / 2\right)}\left(1+\left|\gamma_{\chi}\right|\right)^{\left(\beta_{\chi}+\beta_{\chi^{\prime}}+1 / 2\right)} \\
\leq & \left(1+\left|\gamma_{\chi^{\prime}}\right|\right)^{-\left(\beta_{\chi}+\beta_{\chi^{\prime}}+1 / 2\right)}\left(1+\left|\gamma_{\chi}\right|\right)^{\pi} \\
\leq & \left(1+\left|\gamma_{\chi^{\prime}}\right|\right)^{-\left(\beta_{\chi}+\beta_{\chi^{\prime}}+1 / 2\right)} e^{\pi\left|\gamma_{\chi}\right|}
\end{aligned}
$$

we again obtain $\mathcal{Z}\left(\rho_{\chi}, \rho_{\chi^{\prime}}\right) \ll T^{1 / 2}\left|\gamma_{\chi}\right|^{-1}\left|\gamma_{\chi^{\prime}}\right|^{-1}$. Therefore, the estimate

$$
\mathcal{Z}\left(\rho_{\chi}, \rho_{\chi^{\prime}}\right) \ll T^{1 / 2}\left|\rho_{\chi}\right|^{-1}\left|\rho_{\chi^{\prime}}\right|^{-1}
$$

holds for all $\left|\gamma_{\chi}\right|,\left|\gamma_{\chi^{\prime}}\right| \leq T$ by symmetry between $\rho_{\chi}$ and $\rho_{\chi^{\prime}}$.

By using the estimate (3.15), we have

$$
\sum_{\substack{\rho_{\chi} \\\left|\gamma_{\chi}\right| \leq T}} \sum_{\substack{\rho_{\chi^{\prime}} \\\left|\gamma_{\chi^{\prime}}\right| \leq T}} \mathcal{Z}\left(\rho_{\chi}, \rho_{\chi^{\prime}}\right) x^{\rho_{\chi}+\rho_{\chi^{\prime}}} \ll x^{2 B_{q}} T^{1 / 2} \sum_{\substack{\rho_{\chi} \\\left|\gamma_{\chi}\right| \leq T}} \sum_{\substack{\rho_{\chi^{\prime}} \\\left|\gamma_{\chi^{\prime}}\right| \leq T}}\left|\rho_{\chi}\right|^{-1}\left|\rho_{\chi^{\prime}}\right|^{-1} .
$$

Therefore, by Lemma 2, we have

$$
\begin{aligned}
\Sigma_{3} & \ll x^{2 B_{q}} T^{1 / 2}\left(\frac{1}{\varphi(q)} \sum_{\chi(\bmod q)} \sum_{\substack{\rho_{\chi} \\
\left|\gamma_{\chi}\right| \leq T}} \frac{1}{\left|\rho_{\chi}\right|}\right)^{2} \\
& \ll x^{2 B_{q}} T^{1 / 2}\left((\log q x)^{2}+\frac{q^{1 / 2}(\log q)^{2}}{\varphi(q)}\right)^{2} \ll x^{2 B_{q}} T^{1 / 2}(\log q x)^{4}
\end{aligned}
$$

if $T \leq x$.

All the error terms on the right-hand side of (3.11) have now been estimated. We choose the optimal $T$ by requiring that $x^{2 B_{q}} T^{1 / 2}=x^{2} / T$, hence $T=x^{4\left(1-B_{q}\right) / 3}$. Since $B_{q} \geq 1 / 2$ this choice satisfies the condition $T \leq x$. Substituting this choice of $T$ into (3.11), we obtain the assertion of Proposition 1.

\section{The LAndau-Gonek Formula For $L$-FunCtions}

The Landau-Gonek result [10], originally a formula on the zeros of $\zeta(s)$, has been extended to other situations, for example Ford et al. [7] worked on a general setting of the Selberg class. We did not find any instance where the uniformity with respect to $q$ was treated and we do so here for the sake of completeness.

Proposition 2. Let $x, T, q>1$ and $\chi$ be a primitive character $(\bmod q)$. Then

$$
\begin{aligned}
\sum_{\substack{\rho_{\chi} \\
\left|\gamma_{\chi}\right| \leq T}} x^{\rho_{\chi}}=- & \frac{T}{\pi} \chi(x) \Lambda(x)+O(x(\log 2 q x T)(\log \log 3 x)) \\
& +O\left((\log x) \min \left\{T, \frac{x}{\langle x\rangle}\right\}\right)+O\left((\log 2 q T) \min \left\{T, \frac{1}{\log x}\right\}\right),
\end{aligned}
$$


where $\chi(x)=\Lambda(x)=0$ if $x$ is not an integer, and $\langle x\rangle$ denotes the distance from $x$ to the nearest prime power other than $x$ itself.

Proof. The proof essentially follows the original one of Gonek [10]. Let $c=1+$ $1 / \log 3 x$, and consider the integral

$$
\begin{aligned}
I & =\frac{1}{2 \pi i}\left(\int_{c-i T}^{c+i T}+\int_{c+i T}^{1-c+i T}+\int_{1-c+i T}^{1-c-i T}+\int_{1-c-i T}^{c-i T}\right) \frac{L^{\prime}}{L}(s, \chi) x^{s} d s \\
& =I_{1}+I_{2}+I_{3}+I_{4},
\end{aligned}
$$

say. First suppose that the horizontal paths do not cross any zero of $L(s, \chi)$. The poles inside the rectangle are the non-trivial zeros of $L(s, \chi)$ and at most one trivial zero of $L(s, \chi)$ at $s=0$. Hence the residue theorem gives

$$
I=\sum_{\substack{\rho_{\chi} \\\left|\gamma_{\chi}\right| \leq T}} x^{\rho_{\chi}}+O(1)
$$

We evaluate $I_{1}, I_{2}, I_{3}$ and $I_{4}$. First consider $I_{2}$. We use the well-known formula

$$
\frac{L^{\prime}}{L}(s, \chi)=\sum_{\substack{\rho_{\chi} \\\left|\gamma_{\chi}-t\right| \leq 1}} \frac{1}{s-\rho_{\chi}}+O(\log q(|t|+2))
$$

uniformly in $-1 \leq \sigma \leq 2$ ([14, Lemma 12.6]). We have

$$
I_{2}=\sum_{\substack{\rho_{\chi} \\\left|\gamma_{\chi}-T\right| \leq 1}} \int_{c+i T}^{1-c+i T} \frac{x^{s}}{s-\rho_{\chi}} d s+O\left(\log 2 q T \int_{1-c}^{c} x^{\sigma} d \sigma\right),
$$

whose error term is

$$
\ll x^{c} \log 2 q T \ll x \log 2 q T .
$$

For each integral on the first sum of (4.4), we first observe that $T-1 \leq \gamma_{\chi} \leq T+1$. When $T-1 \leq \gamma_{\chi} \leq T$, we deform the path of integration as

$$
\int_{c+i T}^{1-c+i T}=\int_{c+i T}^{c+i(T+1)}+\int_{c+i(T+1)}^{1-c+i(T+1)}+\int_{1-c+i(T+1)}^{1-c+i T}
$$

Noting that the denominator on the second term is $\gg 1$ and that $\log 3 x \gg 1$, $\log \log 3 x \gg 1$, we obtain in this case

$$
\begin{aligned}
\int_{c+i T}^{1-c+i T} \frac{x^{s}}{s-\rho_{\chi}} d s & \ll x^{c} \int_{T}^{T+1} \frac{d t}{\left|\left(c-\beta_{\chi}\right)+i\left(t-\gamma_{\chi}\right)\right|}+\int_{1-c}^{c} x^{\sigma} d \sigma+\frac{x^{1-c}}{\beta_{\chi}-(1-c)} \\
& \ll x \int_{\gamma_{\chi}}^{\gamma_{\chi}+2} \min \left\{\log 3 x, \frac{1}{t-\gamma_{\chi}}\right\} d t+x+\log 3 x \\
& \ll x\left(\int_{\gamma_{\chi}}^{\gamma_{\chi}+1 / \log 3 x} \log 3 x d t+\int_{\gamma_{\chi}+1 / \log 3 x}^{\gamma_{\chi}+2} \frac{d t}{t-\gamma_{\chi}}\right)+x \\
& \ll x\left(\frac{1}{\log 3 x} \cdot \log 3 x+\left[\log \left(t-\gamma_{\chi}\right)\right]_{t=\gamma_{\chi}+1 / \log 3 x}^{\gamma_{\chi}+2}\right)+x \\
& \ll x \log \log 3 x .
\end{aligned}
$$


If $T<\gamma_{\chi} \leq T+1$, we deform the path to that including the segment with the imaginary part $T-1$, and argue similarly. We can conclude that

$$
I_{2} \ll x \log \log 3 x \sum_{\substack{\rho_{\chi} \\\left|\gamma_{\chi}-T\right| \leq 1}} 1+x \log 2 q T
$$

and hence, by using Lemma 1 ,

$$
I_{2} \ll x(\log 2 q x T)(\log \log 3 x) .
$$

The estimate of $I_{4}$ is similar.

Next consider $I_{3}$. We first quote

$$
\frac{L^{\prime}}{L}(s, \chi)=-\frac{L^{\prime}}{L}(1-s, \bar{\chi})-\log \frac{q}{2 \pi}-\frac{\Gamma^{\prime}}{\Gamma}(1-s)+\frac{\pi}{2} \cot \frac{\pi}{2}(s+\kappa)
$$

([14, (10.35)]), where $\kappa=0$ or 1 depending on whether $\chi$ is an even or an odd character, respectively. We see easily that

$$
\frac{\pi}{2} \cot \frac{\pi}{2}(s+\kappa)= \pm i+O\left(e^{-\pi|t|}\right)
$$

for $|t| \geq 1$ and $\Re s=1-c$. Therefore, putting $s=1-c+i t$ and applying Stirling's formula we have

$$
\frac{L^{\prime}}{L}(1-c+i t, \chi)=-\frac{L^{\prime}}{L}(c-i t, \bar{\chi})-\log q t+C+O\left(t^{-1}\right)
$$

for $t \geq 1$, where $C$ denotes a constant. Hence, the part $[1-c \pm i, 1-c \pm i T]$ of the integral $I_{3}$ is

$$
=\frac{ \pm 1}{2 \pi} \int_{1}^{T}\left(\frac{L^{\prime}}{L}(c \mp i t, \bar{\chi})+\log q t-C\right) x^{1-c \pm i t} d t+O\left(\int_{1}^{T} \frac{x^{1-c}}{t} d t\right),
$$

whose error term is $O(\log T)$. The integral is

$$
=\mp \frac{x^{1-c}}{2 \pi} \sum_{n=2}^{\infty} \frac{\overline{\chi(n)} \Lambda(n)}{n^{c}} \int_{1}^{T}(n x)^{ \pm i t} d t \pm \frac{x^{1-c}}{2 \pi} \int_{1}^{T}(\log q t-C) x^{ \pm i t} d t
$$

whose first part is

$$
\ll x^{1-c} \sum_{n=2}^{\infty} \frac{\Lambda(n)}{n^{c} \log n x} \ll \sum_{n=2}^{\infty} \frac{\Lambda(n)}{n^{c}}=-\frac{\zeta^{\prime}}{\zeta}(c) \ll \frac{1}{c-1} \ll \log 3 x .
$$

The second part is trivially $O(T \log 2 q T)$, while integration by parts gives

$$
=\frac{x^{1-c}}{2 \pi}\left(\left[(\log q t-C) \frac{x^{ \pm i t}}{i \log x}\right]_{t=1}^{T}-\int_{1}^{T} \frac{x^{i t}}{i t \log x} d t\right) \ll \frac{\log 2 q T}{\log x} .
$$

(Note that $\log x \gg 1$ does not hold.) The part $[1-c-i, 1-c+i]$ of the integral $I_{3}$ is $\ll \log 2 q x$ by (4.7). Therefore we conclude

$$
\begin{aligned}
I_{3} & \ll \log 2 q x+(\log 2 q T) \min \left\{T, \frac{1}{\log x}\right\}+\log T \\
& \ll x(\log 2 q T)(\log \log 3 x)+(\log 2 q T) \min \left\{T, \frac{1}{\log x}\right\}
\end{aligned}
$$

since $T>1$. 
We next consider $I_{1}$. Substituting the Dirichlet series expansion, we have

$$
\begin{aligned}
I_{1} & =-\sum_{n=2}^{\infty} \chi(n) \Lambda(n) \frac{1}{2 \pi} \int_{-T}^{T}(x / n)^{c+i t} d t \\
& =-\frac{T}{\pi} \chi(x) \Lambda(x)+O\left(\sum_{n \neq x} \Lambda(n)(x / n)^{c} \min \left\{T, \frac{1}{|\log x / n|}\right\}\right) .
\end{aligned}
$$

The error term here can be estimated by [10, Lemma 2], and so

$$
I_{1}=-\frac{T}{\pi} \chi(x) \Lambda(x)+O(x(\log 2 x)(\log \log 3 x))+O\left((\log x) \min \left\{T, \frac{x}{\langle x\rangle}\right\}\right) .
$$

The formula of the lemma follows by combining (4.6), (4.9) and (4.11).

Finally if the path of $I_{2}$ or $I_{4}$ crosses some zero we choose $T^{\prime}$ slightly larger than $T$, and define $I^{\prime}$, similar to $I$ but now $T$ is replaced by $T^{\prime}$. Then instead of (4.2) we obtain

$$
I^{\prime}=\sum_{\substack{\rho_{\chi} \\\left|\gamma_{\chi}\right| \leq T}} x^{\rho_{\chi}}+O(x \log 2 q T)
$$

(by Lemma 1), while the evaluation of integrals on the edges of the rectangle can be done in the same way as for $I$, so the assertion of the lemma is also valid in this case.

\section{Lemmas For the Proof of Theorem 2}

In this section we present some preparatory material for the improvement of the error term of Proposition 1. We start with a lemma on an integral of the Selbergtype. In order to prove this first lemma, we need to calculate the following sum over the non-trivial zeros of $L(s, \chi)$.

Lemma 6. For any $x \geq 2$ and real number $y$, we have

$$
\sum_{\substack{\rho_{\chi} \\\left|\gamma_{\chi}\right| \leq x}} \frac{1}{1+\left|\gamma_{\chi}-y\right|} \ll(\log q x)^{2} .
$$

Proof. If $|y|>2 x$, then each term above is $\ll x^{-1}$ and the lemma holds trivially. So we consider the case $|y| \leq 2 x$. Then by the triangle inequality, we have

$$
\left|\gamma_{\chi}-y\right| \leq x+y \leq 3 x \text {. }
$$

Thus we can extend the sum and dissect it as

$$
\begin{aligned}
\sum_{\substack{\rho_{\chi} \\
\left|\gamma_{\chi}\right| \leq x}} \frac{1}{1+\left|\gamma_{\chi}-y\right|} & \leq \sum_{\substack{\rho_{\chi} \\
\left|\gamma_{\chi}-y\right| \leq 3 x}} \frac{1}{1+\left|\gamma_{\chi}-y\right|} \\
& \leq \sum_{\substack{\rho_{\chi} \\
\left|\gamma_{\chi}-y\right| \leq 1}} \frac{1}{1+\left|\gamma_{\chi}-y\right|}+\sum_{1 \leq n \leq 3 x} \sum_{\substack{\rho_{\chi} \\
n<\left|\gamma_{\chi}-y\right| \leq n+1}} \frac{1}{1+\left|\gamma_{\chi}-y\right|} \\
& \leq \sum_{\substack{\rho_{\chi} \\
\left|\gamma_{\chi}-y\right| \leq 1}} 1+\sum_{1 \leq n \leq 3 x} \frac{1}{n} \sum_{\substack{\rho_{\chi} \\
n<\left|\gamma_{\chi}-y\right| \leq n+1}} 1 .
\end{aligned}
$$


By using Lemma 1, we can estimate the last sum to be

$$
\ll(\log q x)\left(1+\sum_{1 \leq n \leq 3 x} \frac{1}{n}\right) \ll(\log q x)(\log x) \ll(\log q x)^{2} .
$$

We now obtain an estimate for an integral of the Selberg-type.

Lemma 7. For any $2 \leq h \leq x$ and any $\chi(\bmod q)$, we have

$$
\int_{x}^{2 x}\left|\sum_{t<n \leq t+h} \chi(n) \Lambda(n)-\delta_{0}(\chi) h\right|^{2} d t \ll h x^{2 B_{q}^{*}(\log q x)^{4}} .
$$

Proof. By taking the difference between $u=t+h$ and $u=t$ in Lemma 4, we have

$$
\sum_{t<n \leq t+h} \chi(n) \Lambda(n)=\delta_{0}(\chi) h-\sum_{\substack{\rho_{\chi} \\\left|\gamma_{\chi}\right| \leq T}} \frac{(t+h)^{\rho_{\chi}}-t^{\rho_{\chi}}}{\rho_{\chi}}+E(t+h, T, \chi)-E(t, T, \chi),
$$

where the term $C\left(\chi^{*}\right)$ is cancelled out since it is independent of the main variable $u$ in Lemma 4 . We take the parameter $T=x$. Then the error term is estimated by

$$
E(t+h, T, \chi)-E(t, T, \chi) \ll(\log 2 q)(\log x)+\frac{x}{T}(\log q x T)^{2} \ll(\log q x)^{2} .
$$

Therefore, we obtain

$$
\begin{aligned}
& \sum_{t<n \leq t+h} \chi(n) \Lambda(n)-\delta_{0}(\chi) h \\
= & -\sum_{\substack{\rho_{\chi} \\
\left|\gamma_{\chi}\right| \leq x}} \frac{(t+h)^{\rho_{\chi}}-t^{\rho_{\chi}}}{\rho_{\chi}}+O\left((\log q x)^{2}\right) \\
= & -\sum_{\substack{\rho_{\chi} \\
\left|\gamma_{\chi}\right| \leq x / h}}^{t+h} \int_{t}^{t+h} u^{\rho_{\chi}-1} d u-\sum_{\substack{\rho_{\chi} \\
x / h<\left|\gamma_{\chi}\right| \leq x}} \frac{(t+h)^{\rho_{\chi}}-t^{\rho_{\chi}}}{\rho_{\chi}}+O\left((\log q x)^{2}\right) \\
= & -\int_{t}^{t+h}\left(\sum_{\substack{\rho_{\chi} \\
\left|\gamma_{\chi}\right| \leq x / h}} u^{\rho_{\chi}-1}\right) d u-\sum_{\substack{\rho_{\chi} \\
x / h<\left|\gamma_{\chi}\right| \leq x}} \frac{(t+h)^{\rho_{\chi}}-t^{\rho_{\chi}}}{\rho_{\chi}}+O\left((\log q x)^{2}\right) \\
= & -\psi_{1}(t)-\psi_{2}(t)+O\left((\log q x)^{2}\right), \text { say. }
\end{aligned}
$$

Substituting this explicit formula into the integral of the assertion, we have

$$
\begin{gathered}
\int_{x}^{2 x}\left|\sum_{t<n \leq t+h} \chi(n) \Lambda(n)-\delta_{0}(\chi) h\right|^{2} d t \\
\ll \int_{x}^{2 x}\left|\psi_{1}(t)\right|^{2} d t+\int_{x}^{2 x}\left|\psi_{2}(t)\right|^{2} d t+x(\log q x)^{4} .
\end{gathered}
$$

Hence it suffices to prove the two estimates

$$
\int_{x}^{2 x}\left|\psi_{1}(t)\right|^{2} d t, \quad \int_{x}^{2 x}\left|\psi_{2}(t)\right|^{2} d t \ll h x^{2 B_{q}^{*}}(\log q x)^{4},
$$

since $B_{q}^{*} \geq 1 / 2$ implies $x(\log q x)^{4} \leq h x^{2 B_{q}^{*}}(\log q x)^{4}$. 
18 GAUTAMI BHOWMIK, KARIN HALUPCZOK, KOHJI MATSUMOTO, AND YUTA SUZUKI

Applying the Cauchy-Schwarz inequality to the first integral we have

$$
\left|\psi_{1}(t)\right|^{2} \ll h \int_{t}^{t+h}\left|\sum_{\substack{\rho_{\chi} \\\left|\gamma_{\chi}\right| \leq x / h}} u^{\rho_{\chi}-1}\right|^{2} d u
$$

so that

$$
\begin{aligned}
\int_{x}^{2 x}\left|\psi_{1}(t)\right|^{2} d t & \ll h \int_{x}^{2 x} \int_{t}^{t+h}\left|\sum_{\substack{\rho_{\chi} \\
\left|\gamma_{\chi}\right| \leq x / h}} u^{\rho_{\chi}-1}\right|^{2} d u d t \\
& =h \int_{x}^{2 x+h}\left|\sum_{\substack{\rho_{\chi} \\
\left|\gamma_{\chi}\right| \leq x / h}} u^{\rho_{\chi}-1}\right|^{2}\left(\int_{\max (u-h, x)}^{\min (u, 2 x)} d t\right) d u \\
& \ll h^{2} \int_{x}^{3 x}\left|\sum_{\substack{\rho_{\chi} \\
\left|\gamma_{\chi}\right| \leq x / h}} u^{\rho_{\chi}-1}\right|^{2} d u \ll h^{2} x^{-2} \int_{x}^{3 x}\left|\sum_{\substack{\rho_{\chi} \\
\left|\gamma_{\chi}\right| \leq x / h}} u^{\rho_{\chi}}\right|^{2} d u
\end{aligned}
$$

since $h \leq x$. We now expand the square and integrate over $u$. This gives

$$
\int_{x}^{2 x}\left|\psi_{1}(t)\right|^{2} d t \ll h^{2} x^{-2} \sum_{\substack{\rho_{\chi}, \rho_{\chi}^{\prime} \\\left|\gamma_{\chi}\right|,\left|\gamma_{\chi}^{\prime}\right| \leq x / h}} \frac{x^{\beta_{\chi}+\beta_{\chi}^{\prime}+1}}{1+\left|\gamma_{\chi}-\gamma_{\chi}^{\prime}\right|} .
$$

Using the Vinogradov-Korobov zero-free region (For a detailed proof of a weaker result, see [17, Theorem 1].) and Siegel's theorem, we can estimate $\beta_{\chi}$ and $\beta_{\chi^{\prime}}$ by $B_{q}^{*}$. Therefore,

$$
\begin{aligned}
\int_{x}^{2 x}\left|\psi_{1}(t)\right|^{2} d t & \ll h^{2} x^{2 B_{q}^{*}-1} \sum_{\substack{\rho_{\chi} \\
\left|\gamma_{\chi}\right| \leq x / h}} \sum_{\substack{\rho_{\chi}^{\prime} \\
\left|\gamma_{\chi}^{\prime}\right| \leq x}} \frac{1}{1+\left|\gamma_{\chi}-\gamma_{\chi}^{\prime}\right|} \\
& \ll h^{2} x^{2 B_{q}^{*}-1}(\log q x)^{2} \sum_{\substack{\rho_{\chi} \\
\left|\gamma_{\chi}\right| \leq x / h}} 1 \ll h x^{2 B_{q}^{*}}(\log q x)^{3}
\end{aligned}
$$

by Lemma 6.

For the latter integral, we ignore the difference as

$$
\begin{aligned}
\int_{x}^{2 x}\left|\psi_{2}(t)\right|^{2} d t & \ll \int_{x}^{2 x}\left|\sum_{\substack{\rho_{\chi} \\
x / h<\left|\gamma_{\chi}\right| \leq x}} \frac{(t+h)^{\rho_{\chi}}}{\rho_{\chi}}\right|^{2} d t+\int_{x}^{2 x}\left|\sum_{\substack{\rho_{\chi} \\
x / h<\left|\gamma_{\chi}\right| \leq x}} \frac{t^{\rho_{\chi}}}{\rho_{\chi}}\right|^{2} d t \\
& =\int_{x+h}^{2 x+h}\left|\sum_{\substack{\rho_{\chi} \\
x / h<\left|\gamma_{\chi}\right| \leq x}} \frac{t^{\rho_{\chi}}}{\rho_{\chi}}\right|^{2} d t+\int_{x}^{2 x}\left|\sum_{\substack{\rho_{\chi} \\
x / h<\left|\gamma_{\chi}\right| \leq x}} \frac{t^{\rho_{\chi}}}{\rho_{\chi}}\right|^{2} d t \\
& \ll \int_{x}^{3 x}\left|\sum_{\substack{\rho_{\chi} \\
x / h<\left|\gamma_{\chi}\right| \leq x}} \frac{t^{\rho_{\chi}}}{\rho_{\chi}}\right|^{2} d t .
\end{aligned}
$$


Then we expand the square and integrate over $t$. This gives

$$
\int_{x}^{2 x}\left|\psi_{2}(t)\right|^{2} d t \ll \sum_{\substack{\rho_{\chi}, \rho_{\chi}^{\prime} \\ x / h<\left|\gamma_{\chi}\right|,\left|\gamma_{\chi}^{\prime}\right| \leq x}} \frac{x^{\beta_{\chi}+\beta_{\chi}^{\prime}+1}}{\left|\gamma_{\chi}\right|\left|\gamma_{\chi}^{\prime}\right|\left(1+\left|\gamma_{\chi}-\gamma_{\chi}^{\prime}\right|\right)}
$$

Obviously,

$$
\frac{x^{\beta_{\chi}+\beta_{\chi}^{\prime}}}{\left|\gamma_{\chi}\right|\left|\gamma_{\chi}^{\prime}\right|} \ll \frac{x^{2 \beta_{\chi}}}{\left|\gamma_{\chi}\right|^{2}}+\frac{x^{2 \beta_{\chi}^{\prime}}}{\left|\gamma_{\chi}^{\prime}\right|^{2}}
$$

By using the symmetry of the terms in $\gamma_{\chi}$ and $\gamma_{\chi^{\prime}}$ ) we have

$$
\begin{aligned}
& \int_{x}^{2 x}\left|\psi_{2}(t)\right|^{2} d t \ll x^{1+2 B_{q}^{*}} \sum_{\substack{\rho_{\chi} \\
x / h<\left|\gamma_{\chi}\right| \leq x}} \frac{1}{\left|\gamma_{\chi}\right|^{2}} \sum_{\substack{\rho_{\chi}^{\prime} \\
\left|\gamma_{\chi}^{\prime}\right| \leq x}} \frac{1}{1+\left|\gamma_{\chi}-\gamma_{\chi}^{\prime}\right|}
\end{aligned}
$$

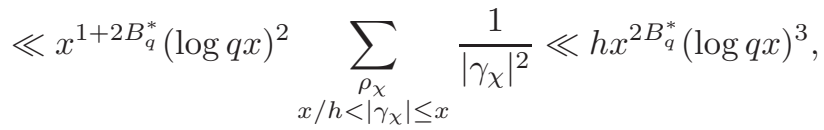

where we used Lemma 3 for the last estimate. This completes the proof.

Now let

$$
T(\alpha)=\sum_{n \leq x} e(n \alpha), \quad S(\alpha, \chi)=\sum_{n \leq x} \chi(n) \Lambda(n) e(n \alpha)
$$

and

$$
W(\alpha, \chi)=S(\alpha, \chi)-\delta_{0}(\chi) T(\alpha)
$$

where $e(\alpha)=\exp (2 \pi i \alpha)$. Our next task is to translate the previous estimate of the integral into this exponential sum setting.

Lemma 8. Let $x^{-1} \leq \xi \leq 1 / 2$. For any $\chi(\bmod q)$, we have

$$
\int_{-\xi}^{\xi}|W(\alpha, \chi)|^{2} d \alpha \ll \xi x^{2 B_{q}^{*}}(\log q x)^{4} .
$$

Proof. We first note that

$$
W(\alpha, \chi)=\sum_{0<n \leq x}\left(\chi(n) \Lambda(n)-\delta_{0}(\chi)\right) e(n \alpha) .
$$

Thus using Gallagher's lemma [9, Lemma 1], we have

$$
\begin{aligned}
\int_{-\xi}^{\xi}|W(\alpha, \chi)|^{2} d \alpha & =\int_{-\xi}^{\xi}\left|\sum_{0<n \leq x}\left(\chi(n) \Lambda(n)-\delta_{0}(\chi)\right) e(n \alpha)\right|^{2} d \alpha . \\
& \ll \xi^{2} \int_{-(2 \xi)^{-1}}^{x}\left|\sum_{a(t)<n \leq b(t)}\left(\chi(n) \Lambda(n)-\delta_{0}(\chi)\right)\right|^{2} d t
\end{aligned}
$$

where

$$
a(t)=\max (t, 0), \quad b(t)=\min \left(t+(2 \xi)^{-1}, x\right) .
$$

We decompose this integral as

$$
\ll \xi^{2} \int_{-(2 \xi)^{-1}}^{(2 \xi)^{-1}}+\xi^{2} \int_{(2 \xi)^{-1}}^{x-(2 \xi)^{-1}}+\xi^{2} \int_{x-(2 \xi)^{-1}}^{x}=\xi^{2} I_{-}+\xi^{2} I+\xi^{2} I_{+}, \text {say. }
$$


By Lemma 4, the Vinogadov-Korobov zero free-region, and Siegel's theorem we obtain

$$
\sum_{n \leq t} \chi(n) \Lambda(n)-\delta_{0}(\chi) t \ll x^{B_{q}^{*}} \sum_{\substack{\rho_{\chi} \neq 1-\beta_{1} \\\left|\gamma_{\chi}\right| \leq x}} \frac{1}{\left|\rho_{\chi}\right|}+\left|\delta_{1}(\chi) \frac{t^{1-\beta_{1}}}{1-\beta_{1}}-C\left(\chi^{*}\right)\right|+(\log q x)^{2}
$$

for $2 \leq t \leq 2 x$. The second term on the right-hand side is estimated by using Theorem 11.4 and formula (12.7) of [14] as

$$
\begin{aligned}
\delta_{1}(\chi) \frac{t^{1-\beta_{1}}}{1-\beta_{1}}-C\left(\chi^{*}\right) & =\delta_{1}(\chi) \frac{t^{1-\beta_{1}}-1}{1-\beta_{1}}+O(\log 2 q) \\
& =\delta_{1}(\chi) \frac{\log t}{1-\beta_{1}} \int_{0}^{1-\beta_{1}} t^{\sigma} d \sigma+O(\log 2 q) \\
& \ll x^{1-\beta_{1}}(\log 2 x)+\log 2 q \ll x^{1 / 2}(\log q x),
\end{aligned}
$$

since $\beta_{1}>1 / 2$. Thus, by Lemma 2 , we have

$$
\sum_{n \leq t} \chi(n) \Lambda(n)-\delta_{0}(\chi) t \ll x^{B_{q}^{*}}(\log q x)^{2}+x^{1 / 2}(\log q x)+(\log q x)^{2} \ll x^{B_{q}^{*}}(\log q x)^{2},
$$

which also holds trivially for $0 \leq t<2$. Thus for any $0 \leq a \leq b \leq 2 x$, we have

$$
\begin{aligned}
\sum_{a<n \leq b}\left(\chi(n) \Lambda(n)-\delta_{0}(\chi)\right) & =\sum_{a<n \leq b} \chi(n) \Lambda(n)-\delta_{0}(\chi)(b-a)+O(1) \\
& \ll x^{B_{q}^{*}}(\log q x)^{2} .
\end{aligned}
$$

By substituting this estimate into $I_{ \pm}$, we obtain

$$
\xi^{2} I_{ \pm} \ll \xi x^{2 B_{q}^{*}}(\log q x)^{4},
$$

since $I_{ \pm}$are integrals taken over intervals of length $\leq \xi^{-1}$. Finally,

$$
\begin{aligned}
\xi^{2} I & =\xi^{2} \int_{(2 \xi)^{-1}}^{x-(2 \xi)^{-1}}\left|\sum_{t<n \leq t+(2 \xi)^{-1}}\left(\chi(n) \Lambda(n)-\delta_{0}(\chi)\right)\right|^{2} d t \\
& \ll \xi^{2} \int_{(2 \xi)^{-1}}^{x}\left|\sum_{t<n \leq t+(2 \xi)^{-1}} \chi(n) \Lambda(n)-\delta_{0}(\chi)(2 \xi)^{-1}\right|^{2} d t+\xi^{2} x \\
& \ll \xi^{2} \sum_{k=0}^{O(\log x)} \int_{x / 2^{k+1}}^{x / 2^{k}}\left|\sum_{t<n \leq t+(2 \xi)^{-1}} \chi(n) \Lambda(n)-\delta_{0}(\chi)(2 \xi)^{-1}\right|^{2} d t+\xi^{2} x \\
& \ll \xi x^{2 B_{q}^{*}(\log q x)^{4}}
\end{aligned}
$$

by Lemma 7. Summing up the above calculations, we obtain the lemma.

Let

$$
J(\chi)=\int_{-1 / 2}^{1 / 2}|W(\alpha, \chi)|^{2}|T(\alpha)| d \alpha .
$$

Now by using the previous results we can obtain an estimate for this quantity.

Lemma 9. We have

$$
J(\chi) \ll x^{2 B_{q}^{*}}(\log q x)^{5} .
$$


Proof. We dissect the integral dyadically as

$$
J(\chi) \leq \int_{|\alpha| \leq 1 / x}|W(\alpha, \chi)|^{2}|T(\alpha)| d \alpha+\sum_{k=1}^{O(\log x)} \int_{1 / 2^{k+1}<|\alpha| \leq 1 / 2^{k}}|W(\alpha, \chi)|^{2}|T(\alpha)| d \alpha .
$$

Then since $T(\alpha) \ll \min \left(x,|\alpha|^{-1}\right)$ for $|\alpha| \leq 1 / 2$, we have

$$
J(\chi) \ll(\log x) \sup _{1 / x<\xi \leq 1 / 2} \xi^{-1} \int_{|\alpha| \leq \xi}|W(\alpha, \chi)|^{2} d \alpha \ll x^{2 B_{q}^{*}}(\log q x)^{5}
$$

by Lemma 8.

\section{Proofs of Theorems 2 And 4}

We let

$$
G\left(n ; \chi_{1}, \chi_{2}\right)=\sum_{\ell+m=n} \chi_{1}(\ell) \Lambda(\ell) \chi_{2}(m) \Lambda(m), \quad S\left(x ; \chi_{1}, \chi_{2}\right)=\sum_{n \leq x} G\left(n ; \chi_{1}, \chi_{2}\right)
$$

and prove the following intermediate lemma.

Lemma 10. For $x \geq 2$ and $\chi_{1}, \chi_{2}(\bmod q)$, we have

$$
\begin{aligned}
& S\left(x ; \chi_{1}, \chi_{2}\right)=\frac{\delta_{0}\left(\chi_{1}\right) \delta_{0}\left(\chi_{2}\right)}{2} x^{2}-\delta_{0}\left(\chi_{2}\right) H\left(x, \chi_{1}\right)-\delta_{0}\left(\chi_{1}\right) H\left(x, \chi_{2}\right)+R\left(x ; \chi_{1}, \chi_{2}\right) \\
& \quad+O\left(\delta_{0}\left(\chi_{2}\right)\left(1+\delta_{1}\left(\chi_{1}\right) q^{1 / 2}\right) x(\log q x)^{2}+\delta_{0}\left(\chi_{1}\right)\left(1+\delta_{1}\left(\chi_{2}\right) q^{1 / 2}\right) x(\log q x)^{2}\right),
\end{aligned}
$$

where

$$
H(x, \chi)=\sum_{\rho_{\chi}} \frac{x^{\rho_{\chi}+1}}{\rho_{\chi}\left(\rho_{\chi}+1\right)}, \quad R\left(x ; \chi_{1}, \chi_{2}\right)=\int_{0}^{1} W\left(\alpha, \chi_{1}\right) W\left(\alpha, \chi_{2}\right) T(-\alpha) d \alpha .
$$

Proof. By the orthogonality of the exponential function we have

$$
S\left(x ; \chi_{1}, \chi_{2}\right)=\int_{0}^{1} S\left(\alpha, \chi_{1}\right) S\left(\alpha, \chi_{2}\right) T(-\alpha) d \alpha .
$$

From the definition we have an expansion

$$
\begin{aligned}
S\left(\alpha, \chi_{1}\right) S\left(\alpha, \chi_{2}\right)=\delta_{0}\left(\chi_{2}\right) S\left(\alpha, \chi_{1}\right) T(\alpha)+\delta_{0}\left(\chi_{1}\right) S\left(\alpha, \chi_{2}\right) T(\alpha) & \\
& -\delta_{0}\left(\chi_{1}\right) \delta_{0}\left(\chi_{2}\right) T(\alpha)^{2}+W\left(\alpha, \chi_{1}\right) W\left(\alpha, \chi_{2}\right) .
\end{aligned}
$$

Substituting this decomposition into the integral expression (6.1), we have

$$
S\left(x ; \chi_{1}, \chi_{2}\right)=\delta_{0}\left(\chi_{2}\right) I\left(\chi_{1}\right)+\delta_{0}\left(\chi_{1}\right) I\left(\chi_{2}\right)-\delta_{0}\left(\chi_{1}\right) \delta_{0}\left(\chi_{2}\right) I+R\left(x ; \chi_{1}, \chi_{2}\right),
$$

where

$$
I=\int_{0}^{1} T(\alpha)^{2} T(-\alpha) d \alpha, \quad I(\chi)=\int_{0}^{1} S(\alpha, \chi) T(\alpha) T(-\alpha) d \alpha .
$$

Therefore it is sufficient to show that

(6.3) $I=\frac{x^{2}}{2}+O(x), \quad I(\chi)=\frac{\delta_{0}(\chi) x^{2}}{2}-H(x, \chi)+O\left(\left(1+\delta_{1}(\chi) q^{1 / 2}\right) x(\log q x)^{2}\right)$.

The first integral $I$ is evaluated by using the orthogonality as

$$
I=\sum_{\ell+m \leq x} 1=\sum_{n \leq x}(n-1)=\frac{x^{2}}{2}+O(x) .
$$


The second integral $I(\chi)$ is

$$
\begin{aligned}
I(\chi) & =\sum_{\ell+m \leq x} \chi(\ell) \Lambda(\ell)=\sum_{n \leq x}(x-n) \chi(n) \Lambda(n)+O(x) \\
& =\sum_{n \leq x} \chi(n) \Lambda(n) \int_{n}^{x} d u+O(x)=\int_{0}^{x} \psi(u, \chi) d u+O(x)
\end{aligned}
$$

by partial summation. We substitute Lemma 5 with $T=x$. Then

$$
I(\chi)=\frac{\delta_{0}(\chi) x^{2}}{2}-\sum_{\substack{\rho_{\chi} \\\left|\gamma_{\chi}\right| \leq x}} \frac{x^{\rho_{\chi}+1}}{\rho_{\chi}\left(\rho_{\chi}+1\right)}+O\left(x(\log q x)^{2}+\delta_{1}(\chi) q^{1 / 2}(\log q)^{2} x\right) .
$$

We then extend the sum over zeros which this gives the error term of the size

$$
\sum_{\substack{\rho_{\chi} \\\left|\gamma_{\chi}\right|>x}} \frac{x^{\rho_{\chi}+1}}{\rho_{\chi}\left(\rho_{\chi}+1\right)} \ll x^{2} \sum_{\substack{\rho_{\chi} \\\left|\gamma_{\chi}\right|>x}} \frac{1}{\left|\rho_{\chi}\right|^{2}} \ll x(\log q x)^{2},
$$

by the use of Lemma 3 in the last estimate. Substituting this estimate into (6.4), we obtain $(6.3)$ for $I(\chi)$.

Now Theorem 2 can be proven.

Proof of Theorem 2. By the orthogonality of characters, we have

$$
S(x ; q, a, b)=\frac{1}{\varphi(q)^{2}} \sum_{\chi_{1}, \chi_{2}(\bmod q)} \overline{\chi_{1}(a) \chi_{2}(b)} S\left(x ; \chi_{1}, \chi_{2}\right) .
$$

Thus, by Lemma 10, it suffices to show that

$$
\frac{1}{\varphi(q)^{2}} \sum_{\chi_{1}, \chi_{2}(\bmod q)}\left|R\left(x ; \chi_{1}, \chi_{2}\right)\right| \ll x^{2 B_{q}^{*}}(\log q x)^{5} .
$$

By the Cauchy-Schwarz inequality and Lemma 9, the right-hand side above is

$$
\ll \frac{1}{\varphi(q)^{2}} \sum_{\chi_{1}, \chi_{2}(\bmod q)} J\left(\chi_{1}\right)^{1 / 2} J\left(\chi_{2}\right)^{1 / 2} \ll x^{2 B_{q}^{*}}(\log q x)^{5} .
$$

This completes the proof.

We move on to the proof of Theorem 4. At first, it might seem that we can obtain this asymptotic formula by summing up Theorem 2 over residues. However this procedure violates the uniformity over $q$ and so instead we take advantage of the "bilinear nature" of the error term $R\left(x ; \chi_{1}, \chi_{2}\right)$ in Lemma 10 . With this in mind we prove the following which will be used in its full generality in the proof of Theorem 3.

Lemma 11. For positive integers $c, q$ and a character $\chi(\bmod q)$, we have

$$
\sum_{\substack{a=1 \\(a(c-a), q)=1}}^{q} \chi(a)=\mu\left(q^{*}\right) \chi^{*}(c) \frac{\varphi(q)}{\varphi\left(q^{*}\right)} \prod_{\substack{p \mid q \\ p \nmid q^{*} c}} \frac{p-2}{p-1},
$$

where $\chi^{*}\left(\bmod q^{*}\right)$ is the primitive character which induces $\chi$. 
Proof. By using the Chinese Remainder Theorem and decomposing the character into the product of characters of prime power moduli it is sufficient to prove the lemma in the case where $q$ is a prime power, say $q=p^{k}$ and $q^{*}=p^{\ell}$. If $\ell=0$, then

$$
\sum_{\substack{a=1 \\(a(c-a), q)=1}}^{q} \chi(a)=\sum_{\substack{a=1 \\(a(c-a), p)=1}}^{p^{k}} 1=\sum_{\substack{a=1 \\ a \neq 0, c(\bmod p)}}^{p^{k}} 1= \begin{cases}p^{k-1}(p-1) & (\text { if } p \mid c), \\ p^{k-1}(p-2) & \text { (if } p \nmid c),\end{cases}
$$

which coincides with the assertion. If $\ell \geq 1$, then we have

$$
\sum_{\substack{a=1 \\(a(c-a), q)=1}}^{q} \chi(a)=\sum_{\substack{a=1 \\(a(c-a), p)=1}}^{p^{k}} \chi^{*}(a)=p^{k-\ell} \sum_{\substack{a=1 \\ a \neq 0, c(\bmod p)}}^{p^{\ell}} \chi^{*}(a) .
$$

If $\ell=1$, then by the orthogonality, this is

$$
=p^{k-1}\left(\sum_{a=1}^{p} \chi^{*}(a)-\sum_{\substack{a=1 \\ a \equiv c(\bmod p)}}^{p} \chi^{*}(a)\right)=\mu(p) \chi^{*}(c) \frac{\varphi\left(p^{k}\right)}{\varphi(p)},
$$

which also coincides with the assertion. If $\ell \geq 2$, then by Theorem 9.4 of [14] we have

$$
\sum_{\substack{a=1 \\(a(c-a), q)=1}}^{q} \chi(a)=p^{k-\ell}\left(\sum_{a=1}^{p^{\ell}} \chi^{*}(a)-\sum_{\substack{a=1 \\ a \equiv c(\bmod p)}}^{p^{\ell}} \chi^{*}(a)\right)=0,
$$

which again satisfies the claimed equality.

Proof of Theorem 4. By using the symmetry between $\ell$ and $m$, we have

$$
\sum_{\substack{n \leq x \\ n \equiv c(\bmod q)}} G(n)=\sum_{\substack{\ell+m \leq x \\ \ell+m \equiv c(\bmod q) \\(\ell m, q)=1}} \Lambda(\ell) \Lambda(m)+O\left(\sum_{\substack{\ell+m \leq x \\ \ell+m \equiv c(\bmod q) \\(m, q)>1}} \Lambda(\ell) \Lambda(m)\right) .
$$

This error term can be estimated as

$$
\sum_{\substack{\ell+m \leq x \\ \ell+m \equiv c(\bmod ) \\(m, q)>1}} \Lambda(\ell) \Lambda(m) \ll \sum_{\ell \leq x} \Lambda(\ell) \sum_{\substack{m \leq x \\(m, q)>1}} \Lambda(m) \ll x(\log q x)^{2},
$$

using the same estimate as in (2.2). Thus it suffices to consider

$$
\sum_{\substack{\ell+m \leq x \\ \ell+m \equiv c(\bmod q) \\(\ell m, q)=1}} \Lambda(\ell) \Lambda(m)=\frac{1}{\varphi(q)^{2}} \sum_{\substack{a=1 \\(a(c-a), q)=1}}^{q} \sum_{\chi_{1}, \chi_{2}(\bmod q)} \overline{\chi_{1}(a) \chi_{2}(c-a)} S\left(x ; \chi_{1}, \chi_{2}\right) .
$$

We apply Lemma 10 to the right-hand side, and evaluate the resulting expression. Clearly,

$$
\frac{1}{\varphi(q)^{2}} \sum_{\substack{a=1 \\(a(c-a), q)=1}}^{q} \sum_{\chi_{1}, \chi_{2}(\bmod q)} \delta_{0}\left(\chi_{2}\right)\left(1+\delta_{1}\left(\chi_{1}\right) q^{1 / 2}\right) x(\log q x)^{2} \ll x(\log q x)^{2} .
$$


Also,

$$
\sum_{\substack{a=1 \\(a(c-a), q)=1}}^{q} 1=\varphi(q)^{2} \mathfrak{S}_{q}(c)
$$

by Lemma 11 with the principal character. Therefore, it suffices to show that

$$
R=\frac{1}{\varphi(q)^{2}} \sum_{\substack{a=1 \\(a(c-a), q)=1}}^{q} \sum_{\chi_{1}, \chi_{2}(\bmod q)} \overline{\chi_{1}(a) \chi_{2}(c-a)} R\left(x ; \chi_{1}, \chi_{2}\right) \ll x^{2 B_{q}^{*}}(\log q x)^{5} .
$$

We have

$$
\begin{gathered}
\sum_{\chi_{1}, \chi_{2}(\bmod q)} \overline{\chi_{1}(a) \chi_{2}(c-a)} R\left(x ; \chi_{1}, \chi_{2}\right) \\
=\int_{0}^{1}\left(\sum_{\chi_{1}(\bmod q)} \overline{\chi_{1}(a)} W\left(\alpha, \chi_{1}\right)\right)\left(\sum_{\chi_{2}(\bmod q)} \overline{\chi_{2}(c-a)} W\left(\alpha, \chi_{2}\right)\right) T(-\alpha) d \alpha .
\end{gathered}
$$

Next the Cauchy-Schwarz inequality gives

$$
R \ll \frac{1}{\varphi(q)^{2}} \sum_{\substack{a=1 \\(a(c-a), q)=1}}^{q} \int_{-1 / 2}^{1 / 2}\left|\sum_{\chi(\bmod q)} \overline{\chi(a)} W(\alpha, \chi)\right|^{2}|T(\alpha)| d \alpha .
$$

By the orthogonality of characters, we have

$$
\begin{aligned}
\sum_{\substack{a=1 \\
(a(c-a), q)=1}}^{q}\left|\sum_{\chi(\bmod q)} \overline{\chi(a)} W(\alpha, \chi)\right|^{2} & \ll \sum_{\substack{a=1 \\
(a, q)=1}}^{q}\left|\sum_{\chi(\bmod q)} \overline{\chi(a)} W(\alpha, \chi)\right|^{2} \\
& =\varphi(q) \sum_{\chi(\bmod q)}|W(\alpha, \chi)|^{2} .
\end{aligned}
$$

Thus, by Lemma 9,

$$
R(\chi) \ll \frac{1}{\varphi(q)} \sum_{\chi(\bmod q)} J(\chi) \ll x^{2 B_{q}^{*}}(\log q x)^{5} .
$$

Summing up the above calculations, we complete the proof.

\section{The connection Between $S(x ; q, a, b)$ And GRH}

Consider the Dirichlet series

$$
F(s)=F(s ; q, a, b)=\sum_{n=1}^{\infty} \frac{G(n ; q, a, b)}{n^{s}},
$$

which converges absolutely and is analytic for $\sigma>2$. Analytic properties of $F(s)$ have been studied by Egami and the third author [6], the first author and SchlagePuchta [4] (in the case $q=1$ ), and by Rüppel [15] (general case). In particular, the connection between $S(x ; q, a, b)$ and GRH can be understood through the analytic continuation of $F(s)$. We first find the meromorphic continuation of $F(s)$ via Theorem 2 in the following proposition. This type of result, under GRH was obtained in $[6],[15]$. 
Proposition 3. The function $F(s)$ can be continued meromorphically to the half plane $\sigma>2 B_{q}$. Its poles in the half plane $\sigma>2 B_{q}$ are

(i) a simple pole at $s=2$ with residue $\varphi(q)^{-2}$,

(ii) a possible pole at $s=\rho_{q}+1$ of at most order 1 with residue

$$
r\left(\rho_{q}\right)=-\frac{1}{\varphi(q)^{2}} \frac{1}{\rho_{q}} \sum_{\substack{\chi(\bmod q) \\ L\left(\rho_{q}, \chi\right)=0}}(\overline{\chi(a)}+\overline{\chi(b)}) m_{\chi}\left(\rho_{q}\right),
$$

where $\rho_{q}$ is a zero of $\prod_{\chi(\bmod q)} L(s, \chi)$ with $0<\Re \rho_{q}<1$ and $m_{\chi}\left(\rho_{q}\right)$ is the multiplicity of $\rho_{q}$ as a zero of $L(s, \chi)$.

In particular, assuming DZC, $B_{q}<1$ and $\bar{\chi}(a)+\bar{\chi}(b) \neq 0$ for all $\chi(\bmod q)$ we obtain

$$
1+B_{q}=\inf \left\{\sigma_{0} \geq \frac{3}{2} \mid F(s)-\frac{1}{\varphi(q)^{2}} \frac{1}{s-2} \text { is analytic on } \sigma>\sigma_{0}\right\} .
$$

Proof. From Theorem 2, we have

$$
S(x ; q, a, b)=\frac{x^{2}}{2 \varphi(q)^{2}}+\sum_{\rho_{q}} r\left(\rho_{q}\right) \frac{x^{\rho_{q}+1}}{\rho_{q}+1}+E(x ; q, a, b)
$$

for $x \geq 1$, where

$$
E(x ; q, a, b) \ll x^{2 B_{q}}(\log 2 q x)^{5}
$$

since $B_{q}^{*} \leq B_{q}$. For $\sigma>2$, we have

$$
F(s)=\int_{1}^{\infty} u^{-s} d S(u ; q, a, b)=s \int_{1}^{\infty} S(u ; q, a, b) u^{-s-1} d u
$$

with integration by parts, because $S(x ; q, a, b)=0$ for $x<4$. Substitute (7.1) in the right-hand side of the above. The swapping of summation and integration is justified due to absolute convergence. Therefore we have

$$
\begin{aligned}
F(s) & =\frac{s}{2 \varphi(q)^{2}(s-2)}+\sum_{\rho_{q}} \frac{r\left(\rho_{q}\right) s}{\left(\rho_{q}+1\right)\left(s-\rho_{q}-1\right)}+s \int_{1}^{\infty} E(u ; q, a, b) u^{-s-1} d u \\
& =\frac{1}{\varphi(q)^{2}(s-2)}+\sum_{\rho_{q}} \frac{r\left(\rho_{q}\right)}{s-\rho_{q}-1}+s \int_{1}^{\infty} E(u ; q, a, b) u^{-s-1} d u+C_{1}(q, a, b),
\end{aligned}
$$

where

$$
C_{1}(q, a, b)=\frac{1}{2 \varphi(q)^{2}}+\sum_{\rho_{q}} \frac{r\left(\rho_{q}\right)}{\rho_{q}+1}
$$

and the sum converges due to Lemma 3 to yield a certain constant depending on $a, b, q$. The sum on the right-hand side of (7.3) converges uniformly for $s \in \mathbb{C} \backslash\left\{\rho_{q}+\right.$ $1\}$ and determines a meromorphic function on $\mathbb{C}$. (Since for $\left|\Im \rho_{q}\right|=\left|\gamma_{\chi}\right|>2|\Im s|$, we have $\left|\rho_{q}+1-s\right| \geq\left|\gamma_{\chi}-\Im s\right| \geq\left|\gamma_{\chi}\right| / 2$, then the compact uniformly convergence can be justified by Lemma 3. Further note that in Lemma 3, each zero appears with multiplicity.) The first and second term on the right-hand side of (7.3) already give 
the announced residues of the proposition. Using the estimate (7.2) we see that the integral

$$
\int_{1}^{\infty} E(u ; q, a, b) u^{-s-1} d u
$$

converges uniformly on the half plane $\sigma>2 B_{q}$ and so it defines an analytic function on $\sigma>2 B_{q}$. This completes the proof of the meromorphic continuation.

For the last assertion, the inequality

$$
1+B_{q} \geq \inf \left\{\sigma_{0} \geq \frac{3}{2} \mid F(s)-\frac{1}{\varphi(q)^{2}} \frac{1}{s-2} \text { is analytic on } \sigma>\sigma_{0}\right\}
$$

follows from the above meromorphic continuation, since $1+B_{q} \geq 2 B_{q}$. We next prove the reverse inequality. If $B_{q}=1 / 2$, then the implication is trivial. Hence we can assume that $1 / 2<B_{q}<1$ and we have $\max \left(2 B_{q}, 3 / 2\right)<1+B_{q}$, so that we can take $\varepsilon>0$ such that $\max \left(2 B_{q}, 3 / 2\right)<1+B_{q}-\varepsilon$. By the definition of $B_{q}$, we can find a zero $\rho_{q}$ such that $1 / 2<B_{q}-\varepsilon<\Re \rho_{q}$. Then by the above meromorphic continuation, we have a possible pole of $F(s)$ of residue $r\left(\rho_{q}\right)$ at $\rho_{q}+1$. (Note that we do not necessarily have meromorphic continuation on $\sigma>1+B_{q}-\varepsilon$ if $B_{q}=1$, since then $B_{q}+1-\varepsilon=2 B_{q}-\varepsilon<2 B_{q}$.) By DZC and the assumption that $\Re \rho_{q}>1 / 2$ we have

$$
r\left(\rho_{q}\right)=-\frac{1}{\varphi(q)^{2}} \frac{1}{\rho_{q}}(\overline{\chi(a)}+\overline{\chi(b)}) m,
$$

where $m \geq 1$. Since we have assumed that $\bar{\chi}(a)+\bar{\chi}(b) \neq 0$ for all $\chi(\bmod q)$, this residue is non-zero so that $\rho_{q}+1$ is a pole of $F(s)$ in the half plane $\sigma>1+B_{q}-\varepsilon>$ $3 / 2$. This implies

$$
1+B_{q}-\varepsilon \leq \inf \left\{\sigma_{0} \geq \frac{3}{2} \mid F(s)-\frac{1}{\varphi(q)^{2}} \frac{1}{s-2} \text { is analytic on } \sigma>\sigma_{0}\right\}
$$

so that on letting $\varepsilon \rightarrow 0$ we obtain the reverse inequality.

We can now prove Theorem 1.

Proof of Theorem 1. First we prove the assertion (1). If $q>x^{\left(1-B_{q}\right) / 2}(\log x)$, then the left-hand side of (1.2) is

$$
\leq\left(\sum_{\substack{\ell \leq x \\ \ell \equiv a(\bmod q)}} \Lambda(\ell)\right)\left(\sum_{\substack{m \leq x \\ m \equiv b(\bmod q)}} \Lambda(m)\right) \ll \frac{(x \log x)^{2}}{q^{2}}+(\log x)^{2} \ll x^{1+B_{q}} .
$$

Also the first term on the right-hand side of (1.2) is $\ll x^{1+B_{q}}$. Thus (1.2) holds trivially. Therefore, we may assume $q \leq x^{\left(1-B_{q}\right) / 2}(\log x)$. By using Lemma 3 ,

$$
\begin{aligned}
& \frac{1}{\varphi(q)^{2}} \sum_{\chi(\bmod q)}(\overline{\chi(a)}+\overline{\chi(b)}) \sum_{\rho_{\chi}} \frac{x^{\rho_{\chi}+1}}{\rho_{\chi}\left(\rho_{\chi}+1\right)} \\
\ll & \frac{x^{B_{q}+1}}{\varphi(q)^{2}} \sum_{\chi(\bmod q)}\left((\log 2 q)^{2}+\delta_{1}(\chi) q^{1 / 2}(\log q)^{2}\right) \ll x^{1+B_{q}} .
\end{aligned}
$$

Therefore, by Theorem 2, we obtain

$$
S(x ; q, a, b)=\frac{x^{2}}{2 \varphi(q)^{2}}+O\left(x^{1+B_{q}}+x^{2 B_{q}^{*}}(\log x)^{5}\right) .
$$


If $1-B_{q}>5 \log \log x / \log x$, then we see that

$$
x^{2 B_{q}^{*}}(\log x)^{5} \leq x^{1+B_{q}} \cdot x^{-\left(1-B_{q}\right)}(\log x)^{5} \leq x^{1+B_{q}}
$$

so that (1.2) follows. Thus we may assume $1-B_{q} \leq 5 \log \log x / \log x$. Further using the assumption on $q$ we find that $q \leq x^{\left(1-B_{q}\right) / 2}(\log x) \leq(\log x)^{7 / 2}$. Thus, by recalling the definition of $\eta=\eta_{q}(x)$ and choosing $\varepsilon=1 / 7$, we obtain

$$
B_{q}^{*} \leq 1-\eta \leq 1-\frac{c_{1}}{\max \left((\log x)^{1 / 2},(\log x)^{4 / 5}\right)}=1-\frac{c_{1}}{(\log x)^{4 / 5}},
$$

where $c_{1}=c_{1}(1 / 7)$ is an absolute constant. This gives

$$
x^{2 B_{q}^{*}}(\log x)^{5} \leq x^{1+B_{q}^{*}}(\log x)^{5} \exp \left(-c_{1}(\log x)^{1 / 5}\right) \ll x^{1+B_{q}} .
$$

Therefore we always arrive at (1.2) .

We next prove the assertion (2). Assume that the formula (1.3) holds, i.e.,

$$
S(x ; q, a, b)=\frac{x^{2}}{2 \varphi(q)^{2}}+E_{d}(x), \quad E_{d}(x) \ll_{q} x^{1+d+\varepsilon}
$$

for arbitrary $\varepsilon>0$. Now we use this formula to obtain the meromorphic continuation of $F(s)$. In the same manner as in the proof of Proposition 3 we have

$$
F(s)-\frac{1}{\varphi(q)^{2}} \frac{1}{s-2}=s \int_{1}^{\infty} E_{d}(u) u^{-s-1} d u+\frac{1}{2 \varphi(q)^{2}}
$$

for $\sigma>2$. Then, by (7.4), the right-hand side gives an analytic function on $\sigma>1+d$. Therefore under the last assertion of Proposition 3, we have $B_{q} \leq d$ provided DZC, $B_{q}<1$ and that $\overline{\chi(a)}+\overline{\chi(b)} \neq 0$ for any $\chi(\bmod q)$. The supplement for $a=b$ is proved in Section 8. This now completes the proof.

We next move on to Theorem 3. The strategy is the same as in the proof of Theorem 1. We consider the Dirichlet series

$$
F_{1}(s)=F_{1}(s ; q ; c)=\sum_{\substack{n=1 \\ n \equiv c(\bmod q)}}^{\infty} \frac{G(n)}{n^{s}} .
$$

The meromorphic continuation of $F_{1}(s)$ is obtained via Theorem 4 .

Proposition 4. The function $F_{1}(s)$ can be continued meromorphically to the half plane $\sigma>2 B_{q}$. Its poles in the half plane $\sigma>2 B_{q}$ are

(i) a possible pole at $s=2$ of order at most 1 with residue $\mathfrak{S}_{q}(c)$,

(ii) a possible pole at $s=\rho_{q}+1$ of at most order 1 with residue

$$
r_{1}\left(\rho_{q}\right)=-\frac{2}{\varphi(q)^{2}} \frac{1}{\rho_{q}} \sum_{\substack{\chi(\bmod q) \\ L\left(\rho_{q}, \chi\right)=0}} m_{\chi}\left(\rho_{q}\right) \sum_{\substack{a=1 \\(a(c-a), q)=1}}^{q} \overline{\chi(a)},
$$

where $\rho_{q}$ is a zero of $\prod_{\chi(\bmod q)} L(s, \chi)$ with $0<\Re \rho<1$.

Proof. This can be proven in the same manner as Proposition 3.

We also require the following lemma. 
Lemma 12. For $x \geq 2$ and positive integers $c, q$, we have

$$
\sum_{\substack{n \leq x \\ n \equiv c(\bmod q)}} J(n)=\frac{\mathfrak{S}_{q}(c)}{2} x^{2}+O(x \log x),
$$

where the implicit constant is absolute.

Proof. We first consider the case of $(2, q) \nmid c$, i.e. $q$ is even while $c$ is odd. Since there is no even number $n \equiv c(\bmod q)$ the sum on the left-hand side is $=0$ since $J(n)=0$ for odd $n$. Also, $\mathfrak{S}_{q}(c)=0$ by definition in this case. Thus the assertion trivially holds for $(2, q) \nmid c$. We next consider the case $(2, q) \mid c$. We use an expression

$$
J(2 N)=2 C_{2} N \sum_{\substack{d \mid N \\ d: \text { odd }}} \frac{\mu(d)^{2}}{\varphi_{2}(d)}, \quad \varphi_{2}(n)=\prod_{p \mid n}(p-2),
$$

to obtain

$$
\sum_{\substack{n \leq x \\ n \equiv c(\bmod q)}} J(n)=\sum_{\substack{2 N \leq x \\ 2 N \equiv c(\bmod q)}} J(2 N)=2 C_{2} \sum_{\substack{d \leq x \\ d: \text { odd }}} \frac{\mu(d)^{2} d}{\varphi_{2}(d)} \sum_{\substack{2 d n \leq x \\ 2 d n \equiv c(\bmod q)}} n .
$$

Let $q_{1}=q /(2 d, q)$. The congruence

$$
2 d n \equiv c(\bmod q)
$$

has a solution, say $c_{1}\left(\bmod q_{1}\right)$ if $(2 d, q) \mid c$, and no solution if $(2 d, q) \nmid c$. Moreover, the condition $(2 d, q) \mid c$ is equivalent to $(d, q) \mid c$ since $d$ is odd and $(2, q) \mid c$. Hence from (7.5), we have

$$
\begin{aligned}
\sum_{\substack{n \leq x \\
n \equiv c(\bmod q)}} J(n) & =2 C_{2} \sum_{\substack{d \leq x / 2 \\
d: \text { odd } \\
(d, q) \mid c}} \frac{\mu(d)^{2} d}{\varphi_{2}(d)} \sum_{\substack{n \leq x / 2 d \\
n \equiv c_{1}\left(\bmod q_{1}\right)}} n \\
& =\frac{C_{2} x^{2}}{4 q} \sum_{\substack{d \leq x / 2 \\
d: \text { odd } \\
(d, q) \mid c}} \frac{\mu(d)^{2}(2 d, q)}{d \varphi_{2}(d)}+O\left(x \sum_{\substack{d \leq x / 2 \\
d: \text { odd }}} \frac{\mu(d)^{2}}{\varphi_{2}(d)}\right) .
\end{aligned}
$$

As for the second term on the right-hand side of (7.6), we have

$$
\sum_{\substack{d \leq x / 2 \\ d: \text { odd }}} \frac{\mu(d)^{2}}{\varphi_{2}(d)} \leq \prod_{2<p \leq x}\left(1+\frac{1}{p-2}\right) \ll \log x .
$$

For the first term on the right-hand side of (7.6), we have

$$
\frac{C_{2}}{4 q} \sum_{\substack{d \leq x / 2 \\
d: \text { odd } \\
(d, q) \mid c}} \frac{\mu(d)^{2}(2 d, q)}{d \varphi_{2}(d)}=\frac{C_{2} \cdot(2, q)}{4 q} \sum_{\begin{array}{c}
d: \text { odd } \\
(d, q) \mid c
\end{array}} \frac{\mu(d)^{2}(d, q)}{d \varphi_{2}(d)}+O\left(\sum_{\substack{d>x / 2 \\
d: \text { odd }}} \frac{\mu(d)^{2}}{d \varphi_{2}(d)}\right)
$$


This remainder term is estimated by using (7.7) as

$$
\sum_{\substack{d>x / 2 \\ d: \text { odd }}} \frac{\mu(d)^{2}}{d \varphi_{2}(d)}=\sum_{\substack{d>x / 2 \\ d: \text { odd }}} \frac{\mu(d)^{2}}{\varphi_{2}(d)} \int_{d}^{\infty} \frac{d u}{u^{2}} \leq \int_{x / 2}^{\infty}\left(\sum_{\substack{d \leq u \\ d: \text { odd }}} \frac{\mu(d)^{2}}{\varphi_{2}(d)}\right) \frac{d u}{u^{2}} \ll \frac{\log x}{x} .
$$

On the other hand we have

$$
\frac{C_{2} \cdot(2, q)}{4 q} \sum_{\substack{d: \text { odd } \\(d, q) \mid c}} \frac{\mu(d)^{2}(d, q)}{d \varphi_{2}(d)}=\frac{C_{2} \cdot(2, q)}{4 q} \prod_{\substack{p>2 \\ p \mid(q, c)}} \frac{p-1}{p-2} \prod_{\substack{p>2 \\ p \nmid q}} \frac{(p-1)^{2}}{p(p-2)} .
$$

Since the definition of $C_{2}$ is

$$
C_{2}=2 \prod_{p>2}\left(1-\frac{1}{(p-1)^{2}}\right)=2 \prod_{p>2} \frac{p(p-2)}{(p-1)^{2}}
$$

the right-hand side of the above is equal to

$$
=\frac{(2, q)}{2 q} \prod_{\substack{p>2 \\ p \mid(q, c)}} \frac{p}{p-1} \prod_{\substack{p>2 \\ p \mid q \\ p \nmid c}} \frac{p(p-2)}{(p-1)^{2}}=\frac{\mathfrak{S}_{q}(c)}{2}
$$

since $(2, q) \mid c$. Substituting (7.9) and (7.10) into (7.8), we have

$$
\frac{C_{2}}{4 q} \sum_{\substack{d \leq x \\ d: \text { odd } \\(d, q) \mid c}} \frac{\mu(d)^{2}(2 d, q)}{d \varphi_{2}(d)}=\frac{\mathfrak{S}_{q}(c)}{2}+O\left(\frac{\log x}{x}\right) .
$$

Combining this with (7.7) and (7.6), we obtain the lemma.

We finally prove Theorem 3 .

Proof of Theorem 3. We first prove (1). By using Lemma 11, the second term on the right-hand side of Theorem 4 is

$$
\begin{aligned}
& =\frac{2}{\varphi(q)^{2}} \sum_{\chi(\bmod q)} \sum_{\rho_{\chi}} \frac{x^{\rho_{\chi}+1}}{\rho_{\chi}\left(\rho_{\chi}+1\right)}\left(\sum_{(a=1}^{q} \overline{\chi(a)}\right) \\
& \ll \frac{x^{B_{q}+1}}{\varphi(q)} \sum_{\chi(\bmod q)} \frac{1}{\varphi\left(q^{*}\right)} \sum_{\rho_{\chi}} \frac{1}{\left|\rho_{\chi}\left(\rho_{\chi}+1\right)\right|} .
\end{aligned}
$$

By Lemma 3, this can be estimated as

$$
\begin{aligned}
\ll x^{1+B_{q}} \frac{q^{2 / 3}}{\varphi(q)} \sum_{\chi(\bmod q)} \frac{1}{\varphi\left(q^{*}\right)} & \ll x^{1+B_{q}} \frac{q^{2 / 3}}{\varphi(q)} \sum_{q^{*} \mid q} \frac{1}{\varphi\left(q^{*}\right)} \sum_{\chi\left(\bmod q^{*}\right)}^{*} 1 \\
& \leq x^{1+B_{q}} \frac{q^{2 / 3} \tau(q)}{\varphi(q)} \ll x^{1+B_{q}}
\end{aligned}
$$

where the summation symbol with $*$ denotes the sum over primitive characters and $\tau(q)$ denotes the number of divisors of $q$. Thus Theorem 4 gives

$$
\sum_{\substack{n \leq x \\ n \equiv c(\bmod q)}} G(n)=\frac{\mathfrak{S}_{q}(c)}{2} x^{2}+O\left(x^{1+B_{q}}+x^{2 B_{q}^{*}}(\log q x)^{5}\right) .
$$


Then an argument similar to that for (1) of Theorem 1 gives (1.9).

We next prove (2). Assume that formula (1.10) holds. Then by Lemma 12,

$$
\sum_{\substack{n \leq x \\ n \equiv c(\bmod q)}} G(n)=\frac{\mathfrak{S}_{q}(c)}{2} x^{2}+E_{d}(x), \quad E_{d}(x) \ll x^{1+d+\varepsilon}
$$

for arbitrary $\varepsilon>0$. As in the case of $F(s)$ we can obtain the meromorphic continuation of $F_{1}(s)$ to the half plane $\sigma>1+d$, which has only one possible pole at $s=2$. We compare this analytic continuation with Proposition 4. By assumption (a) we have $B_{q}=\Re \rho_{0}<1$ so that $2 B_{q}<1+\Re \rho_{0}$. Thus by using assumption (b) $F_{1}(s)$ has a possible pole of order $\leq 1$ with residue

$$
-\frac{2}{\varphi(q)^{2}} \frac{m}{\rho_{0}} \sum_{\substack{a=1 \\(a(c-a), q)=1}}^{q} \overline{\chi(a)}
$$

where $m \geq 1$. By Lemma 11, we find that this residue is non-zero provided under the assumption (c): $q^{*}$ is squarefree, $\left(c, q^{*}\right)=1$, and yet another assumption

$$
2 \nmid q \text { or } 2 \mid q^{*} c,
$$

the last of which is assured by the condition $(2, q) \mid c$ of Theorem 3 . Therefore $\rho_{0}+1$ is a pole of $F_{1}(s)$. By comparing the position of this pole and the analytic continuation we have $1+B_{q} \leq 1+d$. This completes the proof.

\section{ExCLUSiON OF $B_{q}=1$ FOR $a=b$}

In this last section, we exclude the possibility of $B_{q}=1$ for $a=b$ in Theorem 1 (2) following an idea of Ruzsa.

Let $G_{a, q}(n)=G(n ; q, a, a)$, so that $S(x ; q, a, a)=\sum_{n \leq x} G_{a, q}(n)$.

Then our assumption (1.3) in Theorem 1 (2) reads

$$
S(x ; q, a, a)=\frac{x^{2}}{2 \varphi(q)^{2}}+O_{q}\left(x^{1+d+\varepsilon}\right)
$$

for some $1 / 2 \leq d<1$ and any $\varepsilon>0$. We prove that (8.1) together with DZC implies that $B_{q}<1$.

Proof. Step 1. For $|z|<1$ let

$$
F_{a, q}(z)=\sum_{\substack{n \geq 1 \\ n \equiv a(q)}} \Lambda(n) z^{n}, \text { so } F_{a, q}^{2}(z)=\sum_{n \geq 1} G_{a, q}(n) z^{n} .
$$

Then, since $(1-z)^{-1}=1+z+z^{2}+\cdots$, we obtain an identity

$$
\frac{1}{1-z} F_{a, q}^{2}(z)=\sum_{n \geq 1}\left(\sum_{\substack{i+j=n \\ i \geq 1, j \geq 0}} G_{a, q}(i)\right) z^{n}=\sum_{n \geq 1} S(n ; q, a, a) z^{n} .
$$

From (8.1) we deduce that

$$
\frac{1}{1-z} F_{a, q}^{2}(z)=\frac{1}{2 \varphi(q)^{2}} \sum_{n \geq 1} n^{2} z^{n}+O_{q}\left(\sum_{n \geq 1} n^{1+d+\varepsilon}|z|^{n}\right) .
$$


Using the derivative of the geometric series twice we find that

$$
\frac{2}{(1-z)^{3}}=\sum_{n \geq 1} n^{2} z^{n}+O\left(\sum_{n=1}^{\infty} n|z|^{n}\right)
$$

for $|z|<1$ so we can evaluate the main term in (8.2). The above error terms are estimated with the help of the following Lemma.

Lemma 13. For a sequence of positive real numbers $\left(a_{n}\right)_{n=0}^{\infty}$ satisfying

$$
A(x):=\sum_{n \leq x} a_{n} \leq C x^{\kappa}
$$

for all $x \geq 0$ with some constants $C, \kappa \geq 0$, we have

$$
\sum_{n \geq 0} a_{n} e^{-n / X} \leq C \Gamma(\kappa+1) X^{\kappa}
$$

for any real number $X \geq 1$.

Proof. By partial summation and (8.3), the above series is expressed as

$$
\sum_{n \geq 0} a_{n} e^{-n / X}=\frac{1}{X} \int_{0}^{\infty} e^{-u / X} A(u) d u .
$$

Also by (8.3), we estimate this integral by

$$
\leq \frac{C}{X} \int_{0}^{\infty} e^{-u / X} u^{\kappa} d u=C X^{\kappa} \int_{0}^{\infty} e^{-u} u^{\kappa} d u=C \Gamma(\kappa+1) X^{\kappa} .
$$

Thus the lemma follows.

In what follows we work on the circle $|z|=R$ with $R=e^{-1 / N}$ for a large positive integer $N$. Since $\sum_{n \leq x} n^{1+d+\epsilon} \ll x^{2+d+\epsilon}$, by Lemma 13, we continue (8.2) by

$$
\frac{1}{1-z} F_{a, q}^{2}(z)=\frac{1}{\varphi(q)^{2}(1-z)^{3}}+O_{q}\left(N^{C}\right), \quad C:=2+d+\varepsilon<3
$$

on the circle $|z|=R$. Therefore, we obtain

$$
F_{a, q}(z)^{2}=\frac{1}{(1-z)^{2} \varphi(q)^{2}}+O_{q}\left(|1-z| N^{C}\right) .
$$

Note that the second term on the right-hand side is smaller than the first term if

$$
|1-z| \leq c N^{-C / 3}
$$

with sufficiently small constant $c>0$ depending only on $q$ and $d$. Thus on the arc $|z|=R$ with (8.5), which we may call a major arc on $|z|=R$, we can take the complex square root of the formula for $F_{a, q}(z)$ which yields

$$
F_{a, q}(z)= \pm \frac{1}{(1-z) \varphi(q)}+O_{q}\left(|1-z|^{2} N^{C}\right)
$$

as an asymptotic formula for all $z$ on the major arc. Here, the same sign \pm is kept on the whole major arc since $F_{a, q}(z)$ is continuous. Because there are only non-negative coefficients, the left-hand side in (8.6) is non-negative for the choice $z=e^{-1 / N}$. With this choice, the main term in (8.6) is real and therefore must also be non-negative. Therefore, the sign \pm on the right-hand side of (8.6) is + . 
At this point we notice that we are unable to obtain a similar asymptotic formula when $a \neq b$. The square root step here shows that using this method we can prove the exclusion of $B_{q}=1$ in Theorem 1 (2) only when $a=b$.

Step 2. Now we use the kernel

$$
K(z)=z^{-N-1}+z^{-N}+\cdots+z^{-2}=z^{-N-1} \frac{1-z^{N}}{1-z} .
$$

Then by using Cauchy's integral formula, we obtain

$$
\psi(N ; q, a)=\frac{1}{2 \pi i} \int_{|z|=R} F_{a, q}(z) K(z) d z, \quad N=\int_{|z|=R} \frac{1}{1-z} K(z) d z .
$$

Thus we deduce that

$$
\psi(N ; q, a)=\frac{N}{\varphi(q)}+\frac{1}{2 \pi i} \int_{|z|=R}\left(F_{a, q}(z)-\frac{1}{(1-z) \varphi(q)}\right) K(z) d z .
$$

From the second expression of $K(z)$ in (8.7), we see that $K(z) \ll|1-z|^{-1}$. Therefore, on the major arc (8.5) of length $O\left(N^{-C / 3}\right)$ we insert the asymptotic formula (8.6) and the contribution to this integral is $O\left(N^{C-2 C / 3}\right)=O\left(N^{C / 3}\right)$ with $C / 3<1$.

Step 3. For the rest of the circle the minor arc where $|1-z|>c N^{-C / 3}$ we proceed with the Cauchy-Schwarz inequality and apply the Parseval identity.

By the Parseval identity, we obtain the estimate over the full arc

$$
\int_{|z|=R}\left|F_{a, q}(z)-\frac{1}{(1-z) \varphi(q)}\right|^{2} d z \ll \sum_{n \geq 0}(\Lambda(n)+1)^{2} e^{-2 n / N} \ll N^{1+\varepsilon},
$$

where we used the estimate

$$
\sum_{n \leq x}(\Lambda(n)+1)^{2} \ll x \log x \ll x^{1+\varepsilon}
$$

and Lemma 13.

On the other hand, we use the decay of the kernel $K(z)$ on the minor arc. By using the estimate $K(z) \ll|1-z|^{-1}$, we start with

$$
I=\int_{\substack{|z|=R \\|1-z|>c N^{-C / 3}}}|K(z)|^{2} d z \ll \int_{\substack{|z|=R \\|1-z|>c N^{-C / 3}}} \frac{d z}{|1-z|^{2}} .
$$

Now we use the parametrization $z=R e^{i \alpha}=e^{-1 / N+i \alpha}$ with $-\pi \leq \alpha \leq \pi$. On the minor arc, we have

$$
N^{-C / 3} \ll\left|1-e^{-1 / N+i \alpha}\right| \ll\left|-\frac{1}{N}+i \alpha\right| \ll \frac{1}{N}+|\alpha|
$$

so, by recalling the fact that $C<3$, we have $|\alpha| \geq c_{1} N^{-C / 3}$ with some small $c_{1}>0$ depending only on $q$ and $d$. Also, note that

$$
|1-z|^{2}=\left|1-e^{-1 / N} \cos \alpha+i e^{-1 / N} \sin \alpha\right|^{2}=1+e^{-2 / N}-2 e^{-1 / N} \cos \alpha .
$$

By using the inequality of the arithmetic and geometric mean

$$
2\left|e^{-1 / N} \cos \alpha\right| \leq e^{-2 / N}+(\cos \alpha)^{2},
$$

we find that

$$
|1-z|^{2} \geq 1-(\cos \alpha)^{2}=(\sin \alpha)^{2} .
$$


If $|\alpha| \geq \pi / 2$, then $\cos \alpha<0$ so (8.10) implies $|1-z|^{2} \geq 1$. This yields

$$
\begin{aligned}
I \ll \int_{c_{1} N^{-C / 3}<|\alpha| \leq \pi} \frac{d \alpha}{\left|1-R e^{i \alpha}\right|^{2}} & \ll \int_{c_{1} N^{-C / 3}<|\alpha| \leq \pi / 2} \frac{d \alpha}{(\sin \alpha)^{2}}+2 \int_{\pi / 2<|\alpha| \leq \pi} d \alpha \\
& \ll \int_{c_{1} N^{-C / 3}<|\alpha| \leq \pi / 2} \frac{d \alpha}{\alpha^{2}}+1 \ll N^{C / 3} .
\end{aligned}
$$

Putting everything together the Cauchy-Schwarz inequality gives the minor arc estimate for the integral in (8.8) as

$$
\ll\left(\int_{|z|=R}\left|F_{a, q}(z)-\frac{1}{(1-z) \varphi(q)}\right|^{2} d z\right)^{1 / 2} I^{1 / 2} \ll N^{1 / 2+C / 6+\varepsilon}
$$

with $1 / 2+C / 6<1$. This together with the major arc estimate allows us to conclude that

$$
\psi(N ; q, a)-\frac{N}{\varphi(q)} \ll N^{\varepsilon}\left(N^{C / 3}+N^{1 / 2+C / 6}\right) .
$$

The exponent of $N$ is $<1$ for small $\varepsilon>0$. In the explicit formula for

$$
\psi_{a, q}(N)=\frac{1}{\varphi(q)} \sum_{\chi(q)} \bar{\chi}(a) \psi(N, \chi)
$$

that we obtain by inserting the explicit formula for $\psi(N, \chi)$ from Lemma 4 with $T=N$ and assuming DZC, no two terms $N^{\rho_{\chi}} / \rho_{\chi}$ will cancel out for different characters. We conclude that $B_{q}<1$.

\section{REFERENCES}

[1] C. Bauer, Goldbach's conjecture in arithmetic progressions: number and size of exceptional prime moduli, Arch. Math. 108 (2016), 159-172.

[2] G.Bhowmik and I.Z. Ruzsa, Average Goldbach and the Quasi-Riemann Hypothesis, Proceedings of Fourier Analysis and Related Fields, Pécs 2017.

[3] G. Bhowmik and J.-C. Schlage-Puchta, Mean representation number of integers as the sum of primes, Nagoya Math. J. 200 (2010), 27-33.

[4] G. Bhowmik and J.-C. Schlage-Puchta, Meromorphic continuation of the Goldbach generating function, Funct. Approx. Comment. Math. 45 (2011), 43-53.

[5] J. B. Conrey, The Riemann hypothesis, Notices Amer. Math. Soc. 50 (2003), 341-353.

[6] S. Egami and K. Matsumoto, Convolutions of the von Mangoldt function and related Dirichlet series, in "Number Theory. Sailing on the Sea of Number Theory", S. Kanemitsu and J.-Y. Liu (eds.), Ser. Number Theory and Its Appl. 2, World Scientific, 2007, pp.1-23.

[7] K. Ford, K. Soundararajan and A. Zaharescu, On the distribution of imaginary parts of zeros of the Riemann zeta function II, Math. Ann. 343 (2009), 487-505.

[8] A. Fujii, An additive problem of prime numbers, Acta Arith. 58 (1991), 173-179.

[9] P. X. Gallagher, A large sieve density estimate near $\sigma=1$, Invent. Math. 11 (1970), 329-339.

[10] S. M. Gonek, An explicit formula of Landau and its applications to the theory of the zetafunction, Contemp. Math. 143 (1993), 395-413.

[11] A. Granville, Refinements of Goldbach's conjecture, and the generalized Riemann hypothesis, Funct. Approx. Comment. Math. 37 (2007), 159-173; Corrigendum, ibid. 38 (2008), 235-237.

[12] G. H. Hardy and J. E. Littlewood, Some problems of 'Partitio Numerorum'; III: On the expression of a number as a sum of primes, Acta Math. 44 (1923), 1-70.

[13] M.-C. Liu and T. Zhan, The Goldbach problem with primes in arithmetic progressions, in "Analytic Number Theory", Y. Motohashi (ed.), London Math. Soc. Lecture Note Ser. 247, Cambridge Univ. Press, 1997, pp.227-251.

[14] H. L. Montgomery and R. C. Vaughan, Multiplicative Number Theory I, Classical Theory, Cambridge, 2007.

[15] F. Rüppel, Convolutions of the von Mangoldt function over residue classes, Šiauliai Math. Semin. 7 (15) (2012), 135-156. 
34 GAUTAMI BHOWMIK, KARIN HALUPCZOK, KOHJI MATSUMOTO, AND YUTA SUZUKI

[16] Y. Suzuki, A mean value of the representation function for the sum of two primes in arithmetic progressions, Int. J. Number Theory 13 (4) (2017), 977-990.

[17] T. Tatuzawa, On the number of primes in arithmetic progression, Jpn. J. Math. 21 (1951), 93-111.

G. Bhowmik: Laboratoire Paul Painlevé, Labex-Cempi, Université Lille 1, 59655 VilLeneuve d'Asce Cedex, France

E-mail address: bhowmik@math.univ-lille1.fr

K. Halupczok: Mathematisch-Naturwissenschaftliche Fakultät Heinrich-Heine-Universität Düsseldorf, Universitätsstr. 1, 40225 Düsseldorf, Germany

E-mail address: karin.halupczok@uni-duesseldorf.de

K. Matsumoto: Graduate School of Mathematics, Nagoya University, Furocho, ChikusaKU, NAGOYA 464-8602, JAPAN

E-mail address: kohjimat@math.nagoya-u.ac.jp

Y. Suzuki: Graduate School of Mathematics, Nagoya University, Furocho, ChikusaKU, NAGOYA 464-8602, JAPAN

E-mail address: m14021y@math.nagoya-u.ac.jp 